\title{
PATTERN EQUIVARIANT FUNCTIONS, DEFORMATIONS AND EQUIVALENCE OF TILING SPACES
}

\author{
JOHANNES KELLENDONK
}

\begin{abstract}
We reinvestigate the theory of deformations of tilings using $P$-equivariant cohomology. In particular we relate the notion of asymptotically negligible shape functions introduced by Clark and Sadun to weakly $P$-equivariant forms. We then investigate more closely the relation between deformations of patterns and homeomorphism or topological conjugacy of pattern spaces.
\end{abstract}

\section{INTRODUCTION}

The study of aperiodic systems in physics or geometry has led to the definition of cohomology groups associated with aperiodic tilings or point sets of $\mathbb{R}^{n}$ (we use here the word pattern to mean either of them) In physics some elements of these groups are related (via $K$-theory and cyclic cohomology) to topologically quantized transport properties, see KR06 for a recent overview. In geometry Sadun, Williams and Clark have given an interpretation of the cohomology group (with values in $\mathbb{R}^{n}$ ) in terms of deformation theory of tilings [SW03, CS06]. In short, an (admissible) 1-cocycle defines a deformation of a tiling by redefining the shape of its tiles. If the cocycle is a coboundary then the deformed tiling is locally derivable from the original one. A deformation alters the properties of the dynamical system associated with the tiling, except if the new tiling is topologically conjugate to the old one, a notion which is, however, strictly weaker than being mutually locally derivable. To capture also topological conjugacy in cohomological terms, Clark and Sadun introduced the concept of asymptotically negligible cocycles and showed that such cocycles yield deformations which are topologically conjugate.

Clark and Sadun used a formulation of tiling cohomology which is based on the Anderson-Putnam-Gähler construction. This construction furnishes a system of finite $\mathrm{CW}$-complexes so that one can make

Date: June 3, 2022. 
use of their cellular cohomology. Our aim here is to provide a formulation of deformation theory in terms of pattern equivariant functions making use of de Rham cohomology. From this point of view a deformation of a pattern is given by a pattern equivariant 1-form. This allows us to formulate the somewhat ad hoc notion of asymptotically negligible cocycles in terms of natural analytic properties of pattern equivariant functions (Theorem [3.4). In fact, as was introduced in [KP06], there are naturally strongly and weakly pattern equivariant forms on $\mathbb{R}^{n}$ and we find that the theory of deformation modulo topological conjugacy of a pattern $P$ is related to the mixed cohomology group,

$$
Z_{s-P}^{1}\left(\mathbb{R}^{n}, \mathbb{R}^{n}\right) / B_{w-P}^{1}\left(\mathbb{R}^{n}, \mathbb{R}^{n}\right) \cap Z_{s-P}^{1}\left(\mathbb{R}^{n}, \mathbb{R}^{n}\right)
$$

where $Z_{s-P}^{1}\left(\mathbb{R}^{n}, \mathbb{R}^{n}\right)$ denotes the $\mathbb{R}^{n}$-valued strongly $P$-equivariant closed 1 -forms and $B_{w-P}^{1}\left(\mathbb{R}^{n}, \mathbb{R}^{n}\right)$ the $\mathbb{R}^{n}$-valued weakly $P$-equivariant exact 1-forms. A study of the mixed cohomology group for certain classes of tilings is under investigation, for substitution tilings the results of [CS06] point out that this is worthwhile.

As has already been observed by Sadun and Williams, deformations give rise to homeomorphisms between tiling spaces. We review this result in the framework of $P$-equivariant functions. We find that homeomorphisms coming from deformations preserve the canonical transversals and show that this property characterises such homeomorphisms: any homeomorphism between two pattern spaces preserving the canonical transversals and identifying the two patterns comes from a deformation (Cor. 4.11).

We further investigate deformations which give (topologically) conjugate patterns. Our Theorem 4.13 can be seen as the analog of Clark and Sadun's result about asymptotically negligible cocycles: deformations differing from the identity by a weakly $P$-equivariant 1 -form lead to conjugate patterns. More presisely we find that they give rise to a second homeomorphism which also identifies $P$ with its deformed pattern, but does not preserve the canonical transversal. Instead it commutes with the $\mathbb{R}^{n}$-actions. Under an additional assumption on the deformation, which we call boundedness, a converse can be obtained: bounded deformations which yield (pointed) conjugate patterns differ from the identity by a weakly $P$-equivariant 1 -form (Theorem 4.15).

Finally we present a detailed analysis of the question of invertibility of deformations (which somehow is hidden in the notion of admissibility in [CS06]). We show that there exists a neighbourhood of the identity deformation which contains only invertible deformations. 


\section{Preliminaries}

2.1. Pattern spaces and their dynamical systems. The objects we are interested in and which we want somehow to compare are closed subsets of euclidean space, mostly of finite local complexity. These might either be uniformly discrete or they might be tilings; for shortness we call them patterns.

Let $P, Q$ be two closed subsets of $\mathbb{R}^{n}$. Define their distance to be

$$
D(P, Q)=\inf \left\{\epsilon>0 \mid d_{H}\left(B_{\frac{1}{\epsilon}}[P] \cup \partial B\left(0, \frac{1}{\epsilon}\right), B_{\frac{1}{\epsilon}}[Q] \cup \partial B\left(0, \frac{1}{\epsilon}\right)\right) \leq \epsilon\right\} .
$$

Here $B(x, r)$ is the (open) ball of radius $r$ around $x, \partial B(x, r)$ denotes its boundary,

$$
B_{r}[P]=B(0, r) \cap P,
$$

and $d_{H}$ is the Hausdorff distance. We call $B_{r}[P], x \in \mathbb{R}^{n}$, the $r$-patch of $P$ around 0 .

We will mostly look at discrete subsets of $\mathbb{R}^{n}$ but for comparison with CS06 we also look at tilings. There are different ways to associate a discrete point set to a tiling $T$. A possibility would be to introduce a point in the interieur of each tile, in such a way that translationally congruent tiles have that point at the same position. The set of all such points is called the set of punctures of the tiling. We denote by $T^{p t}$. Another possibility which turns out usefull for polyhedral tilings is to look at the set of their vertices, denoted $T^{0}$. The two different ways have their own advantages. For instance, there is a natural bijection between the tiles of $T$ and the points of $T^{p t}$.

$P$ is uniformly discrete if its points have a minimal distance, i.e. if

$$
r_{\text {min }}(P)=\inf \{|x-y|: x \neq y \in P\}
$$

is strictly positive. If $h \in \mathbb{R}^{n}$ and $|h|<\frac{r_{\min }}{2}$ then $D(P, P-h) \leq|h|$.

$P$ is relatively dense if it does not have arbitrarily large holes, i.e. there exists $r$ such that for all $x \in \mathbb{R}^{n}, B_{r}[P-x]$ contains at least one point. A uniformly discrete and relatively dense set is called a Delone set.

For a closed subset $P \subset \mathbb{R}^{n}$ the completion of $\operatorname{orb}(P):=\{P-x \mid x \in$ $\mathbb{R}^{n}$, the $\mathbb{R}^{n}$-orbit of $P$, is called the continuous hull of $P$, written $\Omega_{P}$,

$$
\Omega_{P}:=\overline{\operatorname{orb}(P)}^{D} \text {. }
$$

$\Omega_{P}$ is a compact space which carries a continuous action of $\mathbb{R}^{n}$ induced by the translation action on $P$. We denote the action of $x$ by $\omega \mapsto \omega-x$. This defines the (continuous) dynamical system $\left(\Omega_{P}, \mathbb{R}^{n}\right)$ of $P$. 
If $P$ is uniformly discrete we consider also its discrete hull

$$
\Xi_{P}:=\overline{\{P-p \mid p \in P}^{D} \text {. }
$$

It is a transversal for the $\mathbb{R}^{n}$ action and also referred to as the canonical transversal of $P$. If $T$ is a tiling one may define a transversal and an $r$-discrete groupoid either with $T^{0}$ or with $T^{p t}$. These would differ for the two different choices, but our results below apply to both, provided they are uniformly discrete.

$P$ (or $T$ ) has finite local complexity (w.r.t. the translation group) if for any given $r$ there are only finitely many distinct $r$-patches $B_{r}[P-x]$ with $x \in P\left(\right.$ or $x \in T^{p t}$ or $\left.x \in T^{0}\right)$.

Finite local complexity has strong implications on the structure of the hull. First, all elements of the hull of a discrete pattern $P$ with finite local complexity can be viewed as uniformly discrete subsets of $\mathbb{R}^{n}$ (including, if $P$ is not relatively dense, the empty set). Second, such a hull carries an equivalent but somewhat simpler metric, namely

$$
D_{t}\left(Q, Q^{\prime}\right)=\inf _{r>0}\left\{r^{-1} \mid B_{r}[Q] \cong_{r^{-1}} B_{r}\left[Q^{\prime}\right]\right\},
$$

where we write $B_{r}[Q] \cong_{r^{-1}} B_{r}\left(Q^{\prime}\right)$ for $\exists x, x^{\prime} \in B\left(0, \frac{1}{2 r}\right): B_{r}[Q-x]=$ $B_{r}\left[Q^{\prime}-x^{\prime}\right]$. Restricted to the canonical transversal the metric is even equivalent to

$$
D_{0}\left(Q, Q^{\prime}\right)=\inf _{r>0}\left\{r^{-1} \mid B_{r}[Q]=B_{r}\left[Q^{\prime}\right]\right\} .
$$

Third, the canonical transversal $\Xi_{P}$ is totally disconnected. Finally, at least if $P$ is also relatively dense, the hull is a foliated space whose leaves are locally homeomorphic to $\mathbb{R}^{n}$. To see this let $U_{\epsilon}(Q)$ be the open $\epsilon$ ball around $Q \in \Omega_{P}$ w.r.t. the metric $D_{t}$ and $T_{Q, \epsilon, V}=\left\{Q^{\prime}-v \mid B_{\frac{1}{\epsilon}}\left[Q^{\prime}\right]=\right.$ $\left.B_{\frac{1}{\epsilon}}[Q], v \in V\right\}$ where $V \subset \mathbb{R}^{n}$. Then for any small enough $\epsilon$ one finds $\epsilon_{1}, \epsilon_{2}>0$ such that $U_{\epsilon_{1}}(Q) \subset T_{Q, \epsilon, B(0, \epsilon)} \subset U_{\epsilon_{2}}(Q)$. If $P$ is relatively dense then translates $U_{\epsilon}(Q)-x$ with $Q \in \Xi_{P}$ and $|x|<r_{\max }(P)$ generate the topology and hence the family of sets $T_{Q, \epsilon, B(x, \epsilon)}, Q \in$ $\Xi_{P},|x|<r_{\max }(P), \epsilon<\frac{r_{\min }(P)}{2}$ generates the topology as well. Now $T_{Q, \epsilon, B(x, \epsilon)}$ is the homeomorphic image of $T_{Q, \epsilon,\{0\}} \times B(x, \epsilon)$ under the map $\left(Q^{\prime}, v\right) \mapsto Q^{\prime}-v$. We thus have charts which are Cartesian products of totally disconnected sets (clopen subsets of $\Xi_{P}$ ) with open balls of $\mathbb{R}^{n}$. These are the foliation charts. This has two consequences which will be important further down: First, the path-connected components of $\Omega_{P}$ are the orbits under the translation action, and second, the preimage under the map $\mathbb{R}^{n} \rightarrow \operatorname{orb}(P): x \mapsto P-x$ of the path-connected component of $P$ in $\operatorname{orb}(P) \cap U_{\epsilon}(P)$ lies in $B(0, \epsilon)$. 
2.2. Notions of equivalence. There are a variety of equivalence relations between patterns. Some of them are expressed directly between the patterns, as for instance, mutual local derivability:

Definition 2.1 ( [BSJ91]). Let $P$ and $Q$ be subsets of $\mathbb{R}^{n}$. $Q$ is locally derivable from $P$ if there exists an $R>0$ such that for all $x, y \in \mathbb{R}^{n}$ :

$$
B_{R}[P-x]=B_{R}[P-y] \Longrightarrow\{0\} \cap(Q-x)=\{0\} \cap(Q-y) .
$$

We say that $R$ is a derivability range of the local derivation.

This definition applies to tilings if we regard them as subsets of $\mathbb{R}^{n}$ being given by the boundary points of their tiles.

Equivalently, $Q$ is locally derivable from $P$ if and only if for all $r>0$ exists $r^{\prime}$ such that for all $x, y \in \mathbb{R}^{n}$ :

$$
B_{r^{\prime}}[P-x]=B_{r^{\prime}}[P-y] \Longrightarrow B_{r}[Q-x]=B_{r}[Q-y] .
$$

This has the following interpretation which explains the name: One can construct the $r$-patch of $Q$ around $x$ from the $r^{\prime}$-patch of $P$ around $x$.

Other notions of equivalence refer to the spaces and dynamical systems defined by the patterns. For instance, we could regard patterns as equivalent if they have

(1) homeomorphic hulls, or

(2) topological conjugate (continuous) dynamical systems.

Relation 2 implies relation 1 (trivally). In fact, we will find a stronger relation associated with a deformation, namely an invertible deformation $P^{\prime}$ of $P$ (we explain the notion further down) leads to a homeomorphism between the hulls which preserves the canonical transversal and identifies $P$ with $P^{\prime}$. Likewise we find a relation stronger than 2 , namely we say that $P^{\prime}$ is pointed conjugate to $P$ if there is a topological conjugacy between the (continuous) dynamical systems which maps $P$ to $P^{\prime}$. We will also say that $P^{\prime}$ is pointed semi-conjugate to $P$ if there is a continuous surjection $\Omega_{P} \rightarrow \Omega_{P^{\prime}}$ which commutes with the action of $\mathbb{R}^{n}$ and maps $P$ to $P^{\prime}$. Note that pointed conjugacy implies homeomorphic hulls but the conjugacy will not, in general, preserve the canonical transversal.

2.3. $P$-equivariant functions. Topological spaces can equivalently be decribed by commutative $C^{*}$-algebras. Here these algebras consist of $P$-equivariant functions. For that we need to assume that our subset $P$ has finite local complexity.

Roughly speaking, a function $f$ on $\mathbb{R}^{n}$ is $P$-equivariant with range $R$ if it satisfies the following property: if the patterns in $P$ surrounding 
two points $x$ and $y$ are equal in a ball of radius $R$ (after translating by $-x$ and $-y$, respectively), then $f$ must take the same values at $x$ and $y$. More precisely, we have the following.

Definition 2.2 ([Ke03]). Let $f$ be a function defined on $\mathbb{R}^{n}$. We say that $f$ is $P$-equivariant with range $R$ if for $x, y \in \mathbb{R}^{n}$ :

$$
B_{R}[P-x]=B_{R}[P-y] \Longrightarrow f(x)=f(y) .
$$

$f$ is strongly $P$-equivariant if it is $P$-equivariant with finite range. We also call a function $f$ defined on a subset $Q \subset \mathbb{R}^{n}$ which is locally derivable from $P$ strongly $P$-equivariant if it satisfies the above conditions for $x, y \in Q$.

Note that $Q$ is locally derivable from $P$ if and only if the indicator function on $Q$ is strongly $P$-equivariant.

A priori our definition does not require a strongly $P$-equivariant function to satisfy any further regularity conditions. By adding such conditions we arrive naturally at the concept of weakly $P$-equivariant functions. Let $f: \mathbb{R}^{n} \rightarrow \mathbb{R}^{m}$ and

$$
s_{k}(f)=\sup \left\{\left|D^{\alpha} f(x)\right|: x \in \mathbb{R}^{n},|\alpha| \leq k\right\},
$$

$\alpha=\left(\alpha_{1}, \cdots, \alpha_{n}\right) \in \mathbb{N}^{n}$, where $D^{\alpha} f=\frac{\partial^{\alpha_{1}}}{\partial^{\alpha_{1}} x_{1}} \cdots \frac{\partial^{\alpha_{n}}}{\partial^{\alpha_{n}} x_{n}} f$, and $|\cdot|$ denotes any norm on $\mathbb{R}^{m}$ but $|\alpha|=\sum_{i}^{n} \alpha_{i}$.

Definition 2.3. We denote by $C_{s-P}^{k}\left(\mathbb{R}^{n}, \mathbb{R}^{m}\right), k \in \mathbb{N} \cup\{\infty\}$ the space of strongly $P$-equivariant $C^{k}$-functions over $\mathbb{R}^{n}$ with values in $\mathbb{R}^{m}$. We say that a $C^{k}$-function $f$ is weakly $P$-equivariant if, for all $\epsilon>0$ there exists a strongly $P$-equivariant $C^{k}$-function $f_{\epsilon}$ such that $s_{k}\left(f-f_{\epsilon}\right)<\epsilon$, if $k$ is finite, and $\forall l \in \mathbb{N}: s_{l}\left(f-f_{\epsilon}\right)<\epsilon$, if $k=\infty$. The space of weakly $P$-equivariant $C^{k}$-functions over $\mathbb{R}^{n}$ with values in $\mathbb{R}^{m}$ is denoted $C_{w-P}^{k}\left(\mathbb{R}^{n}, \mathbb{R}^{m}\right)$.

Since strongly $P$-equivariant functions are bounded, $C_{w-P}^{k}\left(\mathbb{R}^{n}, \mathbb{R}^{m}\right)$ is the closure of $C_{s-P}^{k}\left(\mathbb{R}^{n}, \mathbb{R}^{m}\right)$ in the space of functions $f: \mathbb{R}^{n} \rightarrow \mathbb{R}^{m}$ which have bounded continuous derivatives up to order $k$ w.r.t. the topology induced by $s_{k}$, if $k$ is finite, or by all $s_{l}, l \in \mathbb{N}$, if $k=\infty$. Smooth strongly or weakly $P$-equivariant forms can then be identified with elements of $C_{s-P}^{\infty}\left(\mathbb{R}^{n}, \Lambda \mathbb{R}^{n *}\right)$ or $C_{w-P}^{\infty}\left(\mathbb{R}^{n}, \Lambda \mathbb{R}^{n *}\right)$, respectively. As usual, $d f=\sum_{i} \frac{\partial}{\partial x_{i}} f d x_{i}$, and we view $\left\{d x_{i} \mid i=1, \cdots, n\right\}$ as a base for $\mathbb{R}^{n *}$.

Complex-valued continuous weakly $P$-equivariant functions 11 form a $C^{*}$-algebra. The map $f \mapsto f_{P}, f_{P}(x):=f(P-x)$ induces an algebra

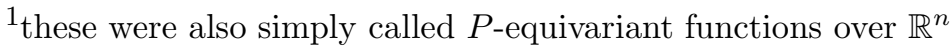


isomorphism between $C\left(\Omega_{P}\right)$ and $C_{w-P}^{0}\left(\mathbb{R}^{n}, \mathbb{C}\right)$. The compactness of $\Omega_{P}$ is reflected in the following lemma whose simple proof is left to the reader:

Lemma 2.4. Let $P$ be a uniformly discrete set with finite local complexity. If $f \in C_{w-P}^{0}\left(\mathbb{R}^{n}, \mathbb{R}^{m}\right)$ then its image is compact. If even $f \in C_{s-P}^{0}\left(\mathbb{R}^{n}, \mathbb{R}^{m}\right)$ then its restriction to $P$ takes only finitely many values.

We now present some general results about $P$-equivariant functions.

Lemma 2.5. Let $P$ be a uniformly discrete set of finite local complexity. Let $\varphi$ and $\psi$ be two differentiable functions which coincide on $P$. Suppose that $d \varphi$ and $d \psi$ are strongly $P$-equivariant. Then $\varphi-\psi$ is strongly P-equivariant.

Proof. Let $\eta=\varphi-\psi$. Hence $\eta$ vanishes on $P$ and $d \eta$ is $P$-equivariant, say with range $R$. Let $r=R+r_{\text {min }}$. Let $B_{r}[P-x]=B_{r}[P-y]$. Choose $p \in P$ such that $p-x \in B_{r_{\text {min }}}[P-x]$. Let $q=p-x+y$. Then $q-y=p-x \in B_{r_{\text {min }}}[P-y]$ and, by $P$-equivariance of $d \eta$,

$$
\eta(x)-\eta(p)=\int_{p}^{x} d \eta=\int_{q}^{y} d \eta=\eta(y)-\eta(q) .
$$

Since $\eta(p)=\eta(q), \eta$ is strongly $P$-equivariant.

Let $f: P \rightarrow \mathbb{R}^{n}$ be a function. For $h=P-P:=\{p-q \mid p, q \in P\}$, we define $\Delta_{h} f: P \cap(P-h) \rightarrow \mathbb{R}^{n}$ by

$$
\Delta_{h} f(x)=f(x+h)-f(x) .
$$

Lemma 2.6. Let $\psi: P \rightarrow \mathbb{R}^{m}$ be a function on a uniformly discrete set $P$ with finite local complexity.

(1) If $\psi$ is strongly $P$-equivariant there exists a smooth strongly $P$ equivariant function $\varphi: \mathbb{R}^{n} \rightarrow \mathbb{R}^{m}$ which restricts on $P$ to $\psi$.

(2) If, for all $\epsilon>0$ there exists a strongly $P$-equivariant function $\psi_{\epsilon}: P \rightarrow \mathbb{R}^{m}$ such that for all $p \in P:\left|\psi(p)-\psi_{\epsilon}(p)\right|<\epsilon$, then there exists a smooth weakly $P$-equivariant function $\varphi: \mathbb{R}^{n} \rightarrow$ $\mathbb{R}^{m}$ which restricts on $P$ to $\psi$.

(3) Suppose that $P$ is moreover relatively dense. If for all $h \in P-P$, the function $\Delta_{h} \psi: P \cap(P-h) \rightarrow \mathbb{R}^{n}$ is strongly $P$-equivariant then there exists a smooth function $\varphi: \mathbb{R}^{n} \rightarrow \mathbb{R}^{m}$ which restricts on $P$ to $\psi$ and has strongly $P$-equivariant differential $d \varphi$.

Proof. Let $\rho: \mathbb{R}^{n} \rightarrow \mathbb{R}$ be a smooth positive function with support contained in $B(0, r), r \leq \frac{r_{\min }}{2}$. For the first two parts of the lemma we 
consider

$$
\varphi(x)=\sum_{p \in P} \rho(x-p) \psi(p)
$$

and suppose that $\rho(0)=1$ so that $\varphi(p)=\psi(p)$ for $p \in P$. If $\psi$ is $P$-equivariant with range $R$ then $\varphi$ is smooth and $P$-equivariant with range $R+\frac{r_{\min }}{2}$.

Suppose that $\psi$ satisfies the conditions of the second part. Then, by the first part, $\varphi_{\epsilon}(x)=\sum_{p \in P} \rho(x-p) \psi_{\epsilon}(p)$ defines a smooth strongly $P$-equivariant function. Now

$$
\left|D^{\alpha}\left(\varphi(x)-\varphi_{\epsilon}(x)\right)\right| \leq\left\|\psi-\psi_{\epsilon}\right\|_{\infty}\left|\sum_{p \in p} D^{\alpha} \rho(x-p)\right| \leq \epsilon\left\|D^{\alpha} \rho\right\|_{\infty}
$$

and hence $\varphi_{\epsilon}$ tends to $\varphi$ in the Fréchet topology when $\epsilon$ tends to 0 .

For the third part consider the Voronoi domains $V_{p}$ for $p \in P$. Let $A$ be an upper bound for their diameter, it is finite by the relative density of $P$. Define for all $x$ in the interior $\operatorname{int}\left(V_{p}\right)$ of the domain

$$
\tilde{\phi}(x):=\psi(p) \text {. }
$$

Then $\tilde{\phi}$ is defined almost everywhere and

$$
\varphi:=\rho * \tilde{\phi}
$$

is a smooth function where we suppose that $\int \rho=1$ so that $\varphi$ coincides with $\psi$ on $P$ provided $r$ is small enough. Now suppose that for all $h=P-P$ with $|h| \leq A, \Delta_{h} \psi$ is $P$-equivariant with range $R>3 A$, and hence, if $B_{R}(P-p)=B_{R}(P-q)$ for some $p, q \in P \cap P-h$ then, for almost all $x \in B(0, R), \tilde{\phi}(x+p+h)-\tilde{\phi}(x+p)=\tilde{\phi}(x+h+q))-\tilde{\phi}(x+q)$. This implies that the equation $\tilde{\phi}(x+p)-\psi(p)=\tilde{\phi}(x+q))-\psi(q)$, which by (2.1) holds for $x \in V_{p}-p$, extends to $x \in B(0,2 A)$. It follows that $\tilde{\phi}(x+p+h)-\psi(p)=\tilde{\phi}(x+h+q)-\psi(q)$ for $x \in B(0,2 A)$ and hence $(d \rho * \tilde{\phi})(x+p+h)=(d \rho *(\tilde{\phi}-\psi(q)+\psi(p))(x+q+h)=(d \rho * \tilde{\phi})(x+q+h)$ where we have used that $d \rho *(\psi(q)-\psi(p))=0$. Since $d \varphi(x)=(d \rho *$ $\tilde{\phi})(x)$ the statement follows.

We finally provide a condition under which a differentiable function with strongly $P$-equivariant differential is weakly $P$-equivariant.

Proposition 2.7. Let $P \in \mathbb{R}^{n}$ be a Delone set of finite local complexity and $\varphi: \mathbb{R}^{n} \rightarrow \mathbb{R}$ be a $C^{k}$-function, $1 \leq k \in \mathbb{N} \cup\{\infty\}$, with strongly $P$-equivariant differential. Then $\varphi$ is weakly $P$-equivariant if and only if for all $\epsilon$ exists a finite partition of $P$ into subsets $P_{1}, \cdots, P_{N}$ which are locally derivable from $P$ and such that $\forall i \in\{1, \cdots, N\} \forall p, q \in P_{i}$ : $|\varphi(p)-\varphi(q)|<\epsilon$. 
Proof. Suppose that $\varphi$ is weakly $P$-equivariant. Choose $\epsilon$ and a strongly $P$-equivariant function $\varphi_{\epsilon}$ such that $\left\|\varphi-\varphi_{\epsilon}\right\|_{\infty}<\epsilon$. In particular $\varphi_{\epsilon}(P)$ is a finite set, lets say $\left\{x_{1}, \cdots, x_{N}\right\}$. Define $P_{i}=\varphi_{\epsilon}^{-1}\left(x_{i}\right) \cap P$ which is certainly locally derivable from $P$. The $P_{i}$ partition $P$ and by construction for all $p, q \in P_{i}$ we have $|\varphi(p)-\varphi(q)|<2 \epsilon$.

We prove the converse: Given $\delta$ choose a finite partition $P_{1}, \cdots, P_{N}$ of sets which are locally derivable from $P$ and such that $\forall i \in\{1, \cdots, N\}$ $\forall p, q \in P_{i}:|\varphi(p)-\varphi(q)|<\delta$. Note that this and the strong $P$ equivariance of $d \varphi$ implies that $\varphi$ has bounded derivatives $D^{\alpha} \varphi,|\alpha| \leq$ $k$. Choose a point $p_{i}$ in each $P_{i}$. Consider the Voronoi domains $V_{p}$ of $p$ in $P$. Define for all $x$ in the interior of $V_{p}$ for $p \in P_{i}$ by $\sigma_{\delta}(x)=\varphi(p)-\varphi\left(p_{i}\right)$. Then $\sigma_{\delta}$ is defined almost everywhere and constant on the interior of the Voronoi domains. Since the $P_{i}$ are locally derivable from $P, \varphi-\sigma_{\delta}$ is strongly $P$-equivariant. It is also $\delta$-close to $\varphi$ where defined and the aim is to smoothen it out.

Let $\rho: \mathbb{R}^{n} \rightarrow \mathbb{R}$ be a smooth positive function with support contained in $B(0,1)$ and $\int \rho=1$. Let $\rho_{\delta}(x)=\delta^{-n} \rho\left(\frac{x}{\delta}\right)$. Define $\varphi_{\delta_{1}, \delta_{2}}=\rho_{\delta_{1}} *(\varphi-$ $\sigma_{\delta_{2}}$ ) which is by construction a smooth strongly $P$-equivariant function. We claim that $\forall \epsilon>0$ and $l \leq k \exists \delta_{1}, \delta_{2}$ such that $s_{l}\left(\left|\varphi-\varphi_{\delta_{1}, \delta_{2}}\right|\right)<\epsilon$. This then proves that we can approximate $\varphi$ in the relevant topology by strongly $P$-equivariant functions.

To proof the claim we fix $\epsilon$ and $l \leq k$. We have

$$
\left\|\rho_{\delta_{1}} * \sigma_{\delta_{2}}\right\|_{\alpha} \leq\left\|\sigma_{\delta_{2}}\right\|_{\infty}\left\|D^{\alpha} \rho_{\delta_{1}}\right\|_{1} \leq \delta_{2} \delta_{1}^{-|\alpha|}\left\|D^{\alpha} \rho\right\|_{1} .
$$

Hence

$$
s_{l}\left(\left|\varphi-\varphi_{\delta_{1}, \delta_{2}}\right|\right) \leq s_{l}\left(\left|\varphi-\rho_{\delta_{1}} * \varphi\right|\right)+\sum_{|\alpha| \leq l} \delta_{2} \delta_{1}^{-|\alpha|}\left\|D^{\alpha} \rho\right\|_{1} .
$$

Since $D^{\alpha} \varphi$ is bounded continuous for $|\alpha| \leq k$ we can find $\delta_{1}$ such that $s_{l}\left(\left|\varphi-\rho_{\delta_{1}} * \varphi\right|\right)<\frac{\epsilon}{2}$ and then $\delta_{2}$ such that $\sum_{|\alpha| \leq l} \delta_{2} \delta_{1}^{-|\alpha|}\left\|D^{\alpha} \rho\right\|_{1}<\frac{\epsilon}{2}$.

2.4. Cohomology groups for patterns and tilings. Let $\mathcal{P}$ be a uniformly discrete subset or a tiling, of finite local complextity. Associated with $\mathcal{P}$ is a cohomology group $H(\mathcal{P}, A)$, the pattern cohomology, with coefficients in an abelian group $A$. It can be described in a variety of equivalent ways:

2.4.1. Cech-cohomology. $H(\mathcal{P}, A)$ is the Cech-cohomology $\check{H}\left(\Omega_{\mathcal{P}}, A\right)$ of the continuous hull.

2.4.2. Cellular cohomology. Let $T$ be a polyhedral tiling, that is a tiling whose tiles are polyhedra and match face to face. We can view $T$ as 
an infinite $\mathrm{CW}$-complex for $\mathbb{R}^{n}$ and suppose to have chosen an orientation of its cells such that translational congruent cells have the same orientation, We also assume that $T$ has finite local complexity. The Anderson-Putnam-Gähler construction expresses $\Omega_{\mathcal{P}}$ as an inverse limit of CW-complexes. We recall the construction: The $k$-neighbourhood of a tile $t$ in a polyhedral tiling $T$ is the patch of all tiles of $T$ which meet the $k-1$-neighbourhood of $t$. Here the 0-neighbourhood of $t$ is simply $t$ itself. A $k$-collared tile is a tile labelled with its $k$-neighbourhood. A $k$-collared prototile is a translational congruence class of a $k$-collared tile. Since $T$ has finite local complexity there are only finitely many $k$-collared prototiles. The $\mathrm{CW}$-complex $\Gamma^{k}$ is the complex obtained from the disjoint union of all $k$-collared prototiles upon identifying any two faces $f_{1}, f_{2}$ of two $k$-collared prototiles $p_{1}, p_{2}$ if one finds in $T$ two tiles $t_{1}, t_{2}$ whose $k$-neighbourhood corresponds to the two $k$-collared prototiles $p_{1}, p_{2}$ such that the faces of $t_{1}$ and $t_{2}$ which correspond to $f_{1}, f_{2}$ agree in $T$. There are surjective maps $\alpha_{k}: \Gamma^{k+1} \rightarrow \Gamma^{k}$ associating to a point in a $k+1$-collared prototile the same point in the $k$-collared prototile obtained by reducing the labelling of the $k+1$-neighbourhood to the $k$-neighbourhood. Then one has the following result [G, Sa03]: $\Omega_{T}$ is the inverse limit of the chain

$$
\cdots \stackrel{\alpha_{k+1}}{\longrightarrow} \Gamma^{k+1} \stackrel{\alpha_{k}}{\longrightarrow} \Gamma^{k} \ldots \stackrel{\alpha_{0}}{\longrightarrow} \Gamma^{0} .
$$

Moreover we have maps $\pi_{k}: T \rightarrow \Gamma^{k}$ associating to a point $x$ in a tile of $T$ the point at the same position in the $k$-collared prototile to which it corresponds.

As a consequence $\check{H}\left(\Omega_{\mathcal{P}}, A\right)$ is the direct limit of the chain

$$
H\left(\Gamma^{0}, A\right) \stackrel{\alpha_{0}^{*}}{\longrightarrow} \cdots H\left(\Gamma^{k}, A\right) \stackrel{\alpha_{k}^{*}}{\longrightarrow} H\left(\Gamma^{k+1}, A\right) \cdots .
$$

Here $H\left(\Gamma^{k}, A\right)$ is the cohomology of CW-complexes. In particular there are homomorphisms $I_{k}: H\left(\Gamma^{k}, A\right) \rightarrow \check{H}\left(\Omega_{\mathcal{P}}, A\right)$ satisfying $I_{k}=I_{k+1} \circ$ $\alpha_{k}^{*}$.

2.4.3. P-equivariant cohomology. The strongly $P$-equivariant forms on $\mathbb{R}^{n}$ form a sub-complex of the de Rham complex for $\mathbb{R}^{n}$. The cohomology of this complex is called the strongly $P$-equivariant cohomology (of $\left.\mathbb{R}^{n}\right)$ and denoted by $H_{s-\mathcal{P}}\left(\mathbb{R}^{n}, \mathbb{R}\right)$. It has been shown in [KP06 that $H_{s-\mathcal{P}}\left(\mathbb{R}^{n}, \mathbb{R}\right)$ is isomorphic to $\check{H}\left(\Omega_{\mathcal{P}}, \mathbb{R}\right)$. This isomorphism has been revisited in Sadun] where the description of $\Omega_{P}$ as inverse limit was used. We provide an explicit description of this isomorphism in degree one.

Let $T$ be a polyhedral tiling of finite local complexity and set $P=T^{0}$. Recall the quotient map $\pi_{k}: T \rightarrow \Gamma^{k}$. Let $e$ be a 1 -cell in $\Gamma^{k}$. Then 
$\pi_{k}^{-1}(e)$ is a union of (oriented) edges of $T$. Suppose that $d \varphi$ is $P$ equivariant with range $r$ and that $r$ is small compared with $k$ (i.e. the tubular $r$-neighbourhood of any tile in $T$ is smaller than its $k$ neighbourhood). Then $\int_{\tilde{e}} d \varphi$ is independent of the choice of $\tilde{e} \in \pi_{k}^{-1}(e)$, since two such preimages agree on their $k$-neighbourhood. Denoting by $Z_{r-P}^{1}\left(\mathbb{R}^{n}, \mathbb{R}^{n}\right)$ the closed $P$-equivariant 1 -forms of range $r$ we may thus define $J_{r}^{k}: Z_{r-P}^{1}\left(\mathbb{R}^{n}, \mathbb{R}^{n}\right) \rightarrow Z^{1}\left(\Gamma^{k}, \mathbb{R}^{n}\right)$ by

$$
J_{r}^{k}(d \varphi)(e)=\int_{\tilde{e}} d \varphi=\varphi(t(\tilde{e}))-\varphi(s(\tilde{e})),
$$

where $s(\tilde{e})$ and $t(\tilde{e})$ are the vertices at which $\tilde{e}$ starts and terminates. It is clear that $J_{r}^{k}(d \varphi)$ is closed, i.e. vanishes on cycles. $J_{r}^{k}(d \varphi)$ is exact if and only if also $\varphi(s(\tilde{e}))$ is independent of the choice of the preimage for $e$ which means that $\varphi$ is strongly $P$-equivariant (with a range with is large compared $k$ but finite). It follows, that $\Theta: H_{s-P}^{1}\left(\mathbb{R}^{n}, \mathbb{R}^{n}\right) \rightarrow$ $\check{H}^{1}\left(\Omega_{P}, \mathbb{R}^{n}\right)$ :

$$
\Theta[d \varphi]=I_{k}\left[J_{r}^{k}(d \varphi)\right]
$$

is well defined. It doesn't depend on the precise choice for $r$ and $k$ as long as $d \varphi$ is $P$-equivariant with range $r$ and $k$ large compared to $r$. Using this flexibility one easily sees that $\Theta$ is additiv and hence defines a group homomorphism.

We show that $\Theta$ is surjective: Let $f \in Z^{1}\left(\Gamma^{k}, \mathbb{R}^{n}\right)$ choose $x_{0} \in T^{0}$ and define $\psi: T^{0} \rightarrow \mathbb{R}^{n}$ by

$$
\psi(t(\tilde{e}))-\psi(s(\tilde{e}))=f\left(\pi_{k}(\tilde{e})\right), \quad \psi\left(x_{0}\right)=0,
$$

for all (oriented) edges $\tilde{e}$ of $T$. Let $h \in \mathbb{R}^{n}$ be the vector corresponding to the edge $\tilde{e}$. Then $\Delta_{h} \psi(x)=\Delta_{h} \psi(y)$ for all $x, y \in T^{0} \cap\left(T^{0}-h\right)$ which have the same $k+1$-neighbourhood. It follows that $\Delta_{h} \psi$ is $P$ equivariant with a range large compared to $k+1$. By Lemma 2.6 Part 3 there exists a smooth function $\varphi$ which coincides with $\psi$ on $P$ and has strongly $P$-equivariant differential. It follows that for large enough $r$ and $l, J_{r}^{l}(d \varphi)=f \circ \alpha_{l-1} \cdots \alpha_{k}$ and hence $\Theta([d \varphi])=I_{k}([f])$. Hence $\Theta$ is surjective. To see that $\Theta$ is injective we note that by (2.2) $J_{r}^{k}(d \varphi)=0$ whenever $\varphi$ is constant on the vertices of the tiling. From Lemma 2.5 it follows then that $\varphi$ is strongly $P$-equivariant, hence $[d \varphi]=0$.

Also the weakly $P$-equivariant forms on $\mathbb{R}^{n}$ form a sub-complex of the de Rham complex for $\mathbb{R}^{n}$ and hence a cohomology group which is called the weakly $P$-equivariant cohomology of $\mathbb{R}^{n}$. We will not consider weakly $P$-equivariant cohomology in this article, but one of our conclusions will be that the mixed group

$$
Z_{s-P}^{1}\left(\mathbb{R}^{n}, \mathbb{R}^{n}\right) / B_{w-P}^{1}\left(\mathbb{R}^{n}, \mathbb{R}^{n}\right) \cap Z_{s-P}^{1}\left(\mathbb{R}^{n}, \mathbb{R}^{n}\right)
$$


is worth further investigation.

\section{ThE FIRST COHOMOLOGY AND DEFORMATIONS}

3.1. The approach of Williams, Sadun and Clark, Sadun. Let $T$ be a polyhedral tiling of finite local complexity with its system of Anderson-Putnam-Gähler complexes $\left(\Gamma^{k}\right)_{k}$.

A shape function is a $\mathbb{R}^{n}$-valued 1-cocycle on $\Gamma^{k}$, i.e. a function $f$ : $\Gamma^{k} \rightarrow \mathbb{R}^{n}$ such that $\delta f(w):=f(\delta w)=0$ for any 2-cell $w$ in $\Gamma^{k}(\delta w$ is the chain corresponding to the boundary of $w$ ). With a little luck a shape function defines a new tiling (up to translation) which is obtained from $T$ by changing the shape of the prototiles. A 1-cell $e$ of $\Gamma^{k}$ is an (oriented) egde of a $k$-collared prototile. $f(e)$ is a vector in $\mathbb{R}^{n}$ and hence defines an oriented straight line segment up to translation which is going to be an edge of a new prototile. Replacing each edge $e$ of a $k$-collared prototile by a line segment parallel to $f(e)$, the condition $\delta f=0$ guaranties that these line segments can be matched together at their boundary points with the same combinatorics as in the prototile. One thus obtaines a new 1-skeleton. Apriori there is no reason that one can complete the new 1-skeleton to a tile by adding in higher dimensional faces in such a way that the combinatorial structure is that of the prototile. But if this is the case, we can construct from $T$, tile by tile, a whole new tiling up to an overall translation, and hence a new tiling space which we denote by $\left(\Omega_{T}\right)_{f}$. It is somewhat clear that all this works if the new edges differ only slightly from the old edges. It then makes sense to speak of a deformation of $T$.

A question which we will investigate in more detail than has been done in CS06] is whether the above process is invertible, i.e. whether one can obtain $T$ back from the new tiling by a similar procedure. Certainly, if the new tiling would be periodic whereas the old one was not, the process could not be inverted. One may argue that invertibility should be no problem if the new edges differ little from the old edges. This is, however, only obvious if $k$ is fixed, since the larger $k$ the more shapes are redefined by the shape function.

Let us call shape functions which allow for all this admissible.

Theorem 3.1 ([SW03, CS06]). Let $f: \Gamma^{0} \rightarrow \mathbb{R}^{n}$ be a cocycle which is admissible. Then $\Omega_{T}$ is homeomorphic to $\left(\Omega_{T}\right)_{f}$.

Theorem 3.2 ([CS06]). Let $f, g: \Gamma^{0} \rightarrow \mathbb{R}^{n}$ be two cocycles which are admissible. If $I_{0}(f)=I_{0}(g)$ then $\left(\Omega_{T}\right)_{f}$ is mutually locally derivable with $\left(\Omega_{T}\right)_{g}$. 
Here the relation of being mutually locally derivable is extended to hulls of tilings by saying that two such hulls are mutually locally derivable if there exists a topological conjugacy between them which maps a tiling to a mutually locally derivable tiling with a uniform derivability range.

Clark and Sadun introduce the notion of asymptotically negligible elements in $H^{1}\left(\Omega_{T}, \mathbb{R}^{n}\right)$. Let us suppose that $r$ is larger than the maximal diameter of a prototile. Then an ordered pair of vertices $\left(x_{1}, x_{2}\right)$ of $T$ is called a recurrence of size $r$ if $B_{r}\left[T-x_{1}\right]=B_{r}\left[T-x_{2}\right]$ but $B_{r+\delta}\left[T-x_{1}\right] \neq B_{r+\delta}\left[T-x_{2}\right]$ for $\delta>0$. If $k$ is small enough so that the $k+1$-neighbourhood of any tile is contained in the $r$-tubular neighbourhood of the tile, then a path between $x_{1}$ and $x_{2}$ along edges defines a loop in the 1-skeleton of $\Gamma^{k}$, different paths leading to homologous loops. An element $\eta \in H^{1}\left(\Gamma^{k}, \mathbb{R}^{n}\right)$ can be evaluated on (the cycle defined by) a loop in $\Gamma^{k}$. It is called asymptotically negligible if for all $\epsilon$ there exists an $r_{\epsilon}$ such that, when $\eta$ is evaluated on a loop in the 1-skeleton of $\Gamma^{k}$ which is defined by a recurrence of size larger than $r_{\epsilon}$, then the result is smaller than $\epsilon$ in norm. The images under the $I_{k}$ of asymptotically negligible elements in $H^{1}\left(\Gamma^{k}, \mathbb{R}^{n}\right)$ are by definition the asymptotic elements of $H^{1}\left(\Omega_{T}, \mathbb{R}^{n}\right)$.

Theorem 3.3 ([CS06]). Let $f, g: \Gamma^{0} \rightarrow \mathbb{R}^{n}$ be two cocycles which are admissible. If $I_{0}(f)-I_{0}(g)$ is asymptotically negligible then $\left(\left(\Omega_{T}\right)_{f}, \mathbb{R}^{n}\right)$ is topologically conjugate to $\left(\left(\Omega_{T}\right)_{g}, \mathbb{R}^{n}\right)$.

It is rather clear that the three theorems extend to shape functions on $\Gamma^{k}$. Note also that, if $f: \Gamma^{k} \rightarrow \mathbb{R}^{n}$ is a shape function then $f \circ$ $\alpha_{k}: \Gamma^{k+1} \rightarrow \mathbb{R}^{n}$ is also a shape function and it leads to the same deformation.

\subsection{Negligible elements versus weakly $P$-equivariant 1 -forms.}

The aim is to reinterprete negligible elements as weakly $P$-equivariant 1 -forms and to interprete Theorem 3.3 in terms of a mixed cohomology group.

Theorem 3.4. An element $\eta \in \check{H}^{1}\left(\Omega_{P}, \mathbb{R}^{n}\right)$ is asymptotically negligible if and only if $\Theta^{-1}(\eta)$ belongs to $B_{w-P}^{1}\left(\mathbb{R}^{n}, \mathbb{R}^{n}\right) \cap Z_{s-P}^{1}\left(\mathbb{R}^{n}, \mathbb{R}^{n}\right) / B_{s-P}^{1}\left(\mathbb{R}^{n}, \mathbb{R}^{n}\right)$.

Proof. Suppose $d \varphi \in B_{w-P}^{1}\left(\mathbb{R}^{n}, \mathbb{R}^{n}\right) \cap Z_{s-P}^{1}\left(\mathbb{R}^{n}, \mathbb{R}^{n}\right)$. Hence for all $\epsilon>0$ exists $R_{\epsilon}$ such that $\varphi=\varphi_{\epsilon}+\psi_{\epsilon}$ where $\varphi_{\epsilon}$ is $P$-equivariant with range $R_{\epsilon}$ and $\left\|\psi_{\epsilon}\right\|_{\infty}<\epsilon$. Also $d \varphi$ is $P$-equivariant with some finite range which we may suppose to be smaller than $R_{\epsilon}$. Let $k$ be large compared to $R_{\epsilon}$ and $r_{\epsilon}$ be large compared to $k+1$. Finally let $\left(x_{1}, x_{2}\right)$ be a recurrence of size larger or equal than $r_{\epsilon}$. Denote by $\left[x_{1}, x_{2}\right]$ the cycle it defines in 
$\Gamma^{k}$. Then we have

$$
J_{R_{\epsilon}}^{k}(d \varphi)\left(\left[x_{1}, x_{2}\right]\right)=\varphi_{\epsilon}\left(x_{2}\right)-\varphi_{\epsilon}\left(x_{1}\right)+\psi_{\epsilon}\left(x_{2}\right)-\psi_{\epsilon}\left(x_{1}\right) .
$$

The first difference drops out since $\left[x_{1}, x_{2}\right]$ is a loop in $\Gamma^{k}$ and so $\left|J_{R_{\epsilon}}^{k}(d \varphi)\left(\left[x_{1}, x_{2}\right]\right)\right|<2 \epsilon$. This shows that $\Theta([d \varphi])$ is asymptotically negligible.

For the converse suppose that $f \in Z^{1}\left(\Gamma^{k}, \mathbb{R}^{n}\right)$ such that $[f]$ is asymptotically negligible. As explained in Section $2.4 .3, \Theta^{-1}([f])$ is represented by the differential of a smooth strongly $P$-equivariant function $\varphi: \mathbb{R}^{n} \rightarrow \mathbb{R}^{n}$ which extends the function $\psi: T^{0} \rightarrow \mathbb{R}^{n}$ defined as in (2.3). Let $\epsilon>0$ and $r_{\epsilon}$ such that $f$ applied to a loop defined by a recurrence $\left(x_{1}, x_{2}\right)$ of size larger than or equal to $r_{\epsilon}$ is smaller in norm than $\epsilon$. By the finite local complexity of $T$ there exist finitely many points $p_{1}, \cdots, p_{f} \in T^{0}$ such that the sets $T_{i}^{0}:=\left\{y \in T^{0} \mid B_{r_{\epsilon}}[T-y]=\right.$ $\left.B_{r_{\epsilon}}\left[T-p_{i}\right]\right\}$ are pairwise disjoint, locally derivable from $T^{0}$, and their union exhausts $T^{0}$. Furthermore, the elements of $\bigcup_{i} T_{i}^{0} \times T_{i}^{0}$ are precisely the recurrences of size larger than or equal to $r_{\epsilon}$. Hence for all $x_{1}, x_{2} \in T_{i}^{0}$ we have $\left|\varphi\left(x_{1}\right)-\varphi\left(x_{2}\right)\right|=\left|f\left(\left[x_{1}, x_{2}\right]\right)\right|<\epsilon$. From Prop. 2.7 follows therefore that $\varphi$ is weakly $P$-equivariant.

\section{Deformations and $P$-equivariant functions}

The aim of this section is to present a theory of deformations which is formulated entirely in the framework of $P$-equivariant functions. We will recover the results of Theorems 3.1,3.2,3.3 (see Theorems 4.8,4.13 and Cor. 4.20) but are able to go further as we obtain refined information about the homeomorphisms involved. As a consequence we are able to decide whether two pattern spaces are homeomorphic (with homeomorphism preserving the abstract transversal) by means of pattern equivariant functions, and to a large extend this turns out to be the case for topological conjugacy as well. Finally we investigate the question of invertibility of deformations.

Our first aim is to interprete the elements entering in the first $P$ equivariant cohomology with values in $\mathbb{R}^{n}$. Since $\mathbb{R}^{n}$ is contractible, any closed 1 -form on $\mathbb{R}^{n}, P$-equivariant or not, can be written as $d \varphi$ for a smooth function $\varphi: \mathbb{R}^{n} \rightarrow \mathbb{R}^{n}$. The first $P$-equivariant cohomology is therefore related to functions, but the restriction that their differential is $P$-equivariant does not imply that they are themself $P$-equivariant which is what makes the cohomology non-trival. For instance $\varphi=$ id is never $P$-equivariant, but $d$ id is.

One may view $\Omega_{P}$ as the compactification of $\mathbb{R}^{n}$ defined by $P \in$ $\mathbb{R}^{n}$. It is then natural to ask how does a function $\varphi: \mathbb{R}^{n} \rightarrow \mathbb{R}^{n}$ affect the compactification, in other words, how does $\Omega_{\varphi(P)}$ compare 
to $\Omega_{P}$ ? Furthermore, one may ask how the pattern dynamical systems compare.

We will often need to make the following assumption about $\varphi$.

Hypothesis 4.1. For all $r>0$ exists $r^{\prime}>0$ such that for all $x \in \mathbb{R}^{n}$ :

$$
B(\varphi(x), r) \cap \varphi(P) \subset \varphi\left(B\left(x, r^{\prime}\right) \cap P\right) .
$$

This assumption is for instance verified if

$$
\forall r>0 \exists r^{\prime}>0 \forall x \in \mathbb{R}^{n}: \quad \varphi^{-1}(B(\varphi(x), r)) \subset B\left(x, r^{\prime}\right) .
$$

Note that if $\varphi$ satisfies (4.1) and $\eta$ is bounded then also $\varphi-\eta$ satisfies (4.1). We say that $\varphi: \mathbb{R}^{n} \rightarrow \mathbb{R}^{n}$ is a bi-Lipschitz map if there exists $\lambda>1$ (the Lipschitz constant) such that

$$
\lambda^{-1}|x-y| \leq|\varphi(x)-\varphi(y)| \leq \lambda|x-y| .
$$

A bi-Lipschitz map is a homeomorphism which satisfies (4.1) with $r^{\prime}=$ $\lambda r$. In fact, it is injective and continuous, and hence has open image by Brouwer's theorem. Moreover its image is also closed since a pre-image of a Cauchy sequence is a Cauchy sequence, and hence it is surjective. A further advantage of a bi-Lipschitz map is that it maps Delone sets to Delone sets. We are particularily interested in the case $\varphi=\mathrm{id}+\eta$ where $\eta$ is differentiable and $\|d \eta\|_{\infty}<1$ (or $\varphi=g+\eta$ with $g \in G L(n, \mathbb{R})$ and $\left.\|d \eta\|_{\infty}<\left(\left\|g^{-1}\right\|_{\infty}\right)^{-1}\right)$. Such a map $\varphi$ is a bi-Lipschitz map with $\lambda^{-1}=1-\|d \eta\|_{\infty}$.

Lemma 4.2. Let $P$ be a uniformly discrete set of finite local complexity. Let $\varphi: \mathbb{R}^{n} \rightarrow \mathbb{R}^{n}$ be a function, $g \in G L(n, \mathbb{R})$ and $\varphi-g$ be $P$-equivariant with range $R$. Then we have for all $r>0$ and $x, y \in \mathbb{R}^{n}$ :

$$
\begin{aligned}
& B_{R+r}[P-x]=B_{R+r}[P-y] \Longrightarrow \\
& \quad \forall h \in B(0, r): \varphi(x+h)-g(x)=\varphi(y+h)-g(y) .
\end{aligned}
$$

In particular

$$
B_{R+r}[P-x]=B_{R+r}[P-y] \Longrightarrow B_{r}(\varphi(P)-g(x))=B_{r}(\varphi(P)-g(y))
$$

and $\varphi(P)$ is locally derivable from $g(P)$.

Proof. By assumption $B_{R+r}[P-x]=B_{R+r}[P-y]$ implies $\forall h \in B(0, r)$ : $\varphi(x+h)-g(x+h)=\varphi(y+h)-g(y+h)$. The statement follows therefore from the linearity of $g$. The last statement is trivial for $g=\mathrm{id}$. Now if $\varphi-g$ is strongly $P$-equivariant then $\varphi \circ g^{-1}-$ id is strongly $g(P)$ equivariant. This implies the last statement for general $g$.

Note that $d(\varphi-g)$ is strongly $P$-equivariant whenever $d \varphi$ is strongly $P$-equivariant. 
Lemma 4.3. Let $P$ be a uniformly discrete set of finite local complexity and $\varphi: \mathbb{R}^{n} \rightarrow \mathbb{R}^{n}$ be a differentiable function. If $d \varphi$ is $P$-equivariant with range $R$ then for all $x, y \in \mathbb{R}^{n}$ :

$$
\begin{aligned}
& B_{R+r}[P-x]=B_{R+r}[P-y] \Longrightarrow \\
& \quad \forall h \in B(0, r): \varphi(x+h)-\varphi(x)=\varphi(y+h)-\varphi(y) .
\end{aligned}
$$

If moreover $\varphi$ satisfies Hypothesis 4.1 then for all $r>0$ exists $r^{\prime}$ such that

$$
B_{r^{\prime}}[P-x]=B_{r^{\prime}}[P-y] \Longrightarrow B_{r}(\varphi(P)-\varphi(x))=B_{r}(\varphi(P)-\varphi(y)) .
$$

Proof. Let $B_{R+r}[P-x]=B_{R+r}[P-y]$ and $h \in B_{r}$. As $d \varphi$ is $P$ equivariant with range $R$ we have $d \varphi(x+h)=d \varphi(y+h)$. In particular $\int_{x}^{x+h} d \varphi=\int_{y}^{y+h} d \varphi$ (we can integrate along a straight line). As $\varphi(x+$ $h)-\varphi(x)=\int_{x}^{x+h} d \varphi$ the first statement holds true.

Suppose $B_{R+r^{\prime}}[P-x]=B_{R+r^{\prime}}[P-y]$. According to the first statement this implies

$$
\varphi\left(B\left(x, r^{\prime}\right) \cap P\right)-\varphi(x)=\varphi\left(B\left(y, r^{\prime}\right) \cap P\right)-\varphi(y) .
$$

Now let $r$ be given and choose $r^{\prime}$ according to Hypothesis 4.1. Then, for any $z \in \mathbb{R}^{n},\left(\varphi\left(B\left(z, r^{\prime}\right) \cap P\right)-\varphi(z)\right) \cap B(0, r)=\left(\varphi\left(B\left(z, r^{\prime}\right) \cap\right.\right.$ $P) \cap B(\varphi(z), r))-\varphi(z)=(\varphi(P) \cap B(\varphi(z), r))-\varphi(z)$. The latter is by definition $B_{r}[\varphi(P)-\varphi(z)]$ and so (4.3) implies $B_{r}[\varphi(P)-\varphi(x)]=$ $B_{r}[\varphi(P)-\varphi(y)]$.

Corollary 4.4. Let $P$ be a uniformly discrete set of finite local complexity. Let $\varphi: \mathbb{R}^{n} \rightarrow \mathbb{R}^{n}$ be a differentiable function which fullfills Hypothesis 4.1. If $d \varphi$ is strongly P-equivariant then $\varphi(P)$ has finite local complexity.

Proof. Finite local complexity of $P$ means that there are for any given $r^{\prime}$ only finitely many different $B\left(0, r^{\prime}\right) \cap(P-x), x \in P$. Thus we conclude from Lemma 4.3 that there are also only finitely many different $B(0, r) \cap$ $(\varphi(P)-z), z \in \varphi(P)$.

There is no reason for $\varphi(P)$ to be of finite local complexity if we require merely weak $P$-equivariance of $d(\varphi-g)$ or $d \varphi$.

How can we interprete (4.2)? Clearly it allows to construct the $r$ patch of $\varphi(P)$ around $\varphi(x)$ from the $r^{\prime}$-patch of $P$ around $x$, but this involves the passage from $\varphi(x)$ to $x$ which is apriori not local (i.e. not determined by a patch of $P$ ). Nevertheless, if we forget about the position of the patches in $\mathbb{R}^{n}$ and consider their translational congruence classes we obtain from (4.2) a local map: to each translational congruence class of an $r$-patch of $P$ is associated a unique translational 
congruence class of an $r^{\prime}$-patch of $\varphi(P)$. In one dimension this gives a rewriting rule. In higher dimensions one obtains a local derivation in the sense of [Ke97]. If $\varphi=\mathrm{id}+\eta$ with small $\|d \eta\|_{\infty}$ and $P$ is the set of vertices of a polyhedral tiling the local map defined by (4.2) can be interpreted as a deformation of the tiling induced by changing the length of the edges of its tiles [SW03], see Sect. 3.1. In view of this and the results in Sect. 2.4.3 we define:

Definition 4.5. Let $P$ be a Delone set in $\mathbb{R}^{n}$. A Delone set $P^{\prime} \subset \mathbb{R}^{n}$ is a deformation of $P$ if there exists a smooth function $\varphi: \mathbb{R}^{n} \rightarrow \mathbb{R}^{n}$ satisfying Hypothesis 4.1 and having a strongly $P$-equivariant differential such that $P^{\prime}=\varphi(P)$.

We have a lot of freedom to choose $\varphi$. In fact, since the joint kernel of the maps $J_{r}^{k}$ from Sect. 2.4.3 is

$$
N_{s-P}^{1}\left(\mathbb{R}^{n}, \mathbb{R}^{n}\right)=\left\{d \eta \in B_{s-P}^{1}\left(\mathbb{R}^{n}, \mathbb{R}^{n}\right) \mid \eta \text { is constant on } P\right\}
$$

the elements of $Z_{s-P}^{1}\left(\mathbb{R}^{n}, \mathbb{R}^{n}\right) / N_{s-P}^{1}\left(\mathbb{R}^{n}, \mathbb{R}^{n}\right)$ parametrise the deformations of $P$ (or of a tiling $T$ with $P=T^{0}$ ).

We end this section with the following important definition, which is inspired from the above lemmata.

Definition 4.6. Let $P$ be a uniformly discrete set of finite local complexity.

(1) Let $\varphi: \mathbb{R}^{n} \rightarrow \mathbb{R}^{n}$ be a $C^{0}$-function such that $\varphi-g$ is weakly $P$-equivariant. We define $\Phi_{g}: \operatorname{orb}(P) \rightarrow \Omega_{\varphi(P)}$,

$$
\Phi_{g}(P-x):=\varphi(P)-g(x) .
$$

(2) Let $\varphi: \mathbb{R}^{n} \rightarrow \mathbb{R}^{n}$ be a differentiable function such that $d \varphi$ is strongly $P$-equivariant. We define $\Phi_{\varphi}: \operatorname{orb}(P) \rightarrow \Omega_{\varphi(P)}$,

$$
\Phi_{\varphi}(P-x):=\varphi(P)-(\varphi(x)-\varphi(0)) .
$$

That these maps are well-defined is the issue of the following lemma.

Lemma 4.7. : We assume the notation and conditions of the last definition. In the first case $P-x=P$ implies $\varphi(P)-g(x)=\varphi(P)$. In the second case $P-x=P$ implies $\varphi(P)-\varphi(x)=\varphi(P)-\varphi(0)$.

Proof. Suppose that $\varphi-g$ is strongly $P$-equivariant. By Lemma 4.2 $\forall h \in P: \varphi(-x+h)-g(-x)=\varphi(h)$ which implies $\varphi(P)=\varphi(P)-g(x)$. If $\varphi-g$ is merely weakly $P$-equivariant we can find for all $\epsilon>0$ an approximation $\varphi_{\epsilon}$ of $\varphi$ such that $\varphi_{\epsilon}-g$ is strongly $P$-equivariant and $\left\|\varphi_{\epsilon}-\varphi\right\|_{\infty}<\epsilon$. The above then implies that $d_{h}(\varphi(P)-g(x), \varphi(P))<$ $2 \epsilon$. 
Now suppose that $d \varphi$ is strongly $P$-equivariant. By Lemma $4.3 \forall h \in$ $P: \varphi(x+h)-\varphi(x)=\varphi(h)-\varphi(0)$. This implies $\varphi(P+x)=\varphi(P)+$ $\varphi(x)-\varphi(0)$ and hence the statement.

In the following we shall see that $\Phi_{g}$ and $\Phi_{\varphi}$ can be extended to maps between $\Omega_{P}$ and $\Omega_{\varphi(P)}$, the second only in the strongly $P$-equivariant case. This is the case once we can show that they are uniformly continuous, since a uniformly continuous map between (not neccessarily complete) metric spaces has a unique continuous extension to the completions.

\subsection{Closed strongly $P$-equivariant 1 -forms and deformations.}

We investigate more closely $P$-equivariant 1 -forms, that is $P$-equivariant differentials, and how they affect the tiling spaces and dynamical systems. We start by the observation that a strongly $P$-equivariant 1 -form gives rise to a continuous map between hulls of Delone sets preserving the canonical transversal, an observation which is slightly stronger than Theorem 3.1.

Theorem 4.8. Let $P$ be a Delone set of finite local complexity. Let $\varphi: \mathbb{R}^{n} \rightarrow \mathbb{R}^{n}$ be a $C^{1}$-map which fullfills Hypothesis 4.1 and whose differential $d \varphi$ is strongly $P$-equivariant. Then $\Phi_{\varphi}$ is uniformly continuous map. It thus extends to a continuous map $\Phi_{\varphi}: \Omega_{P} \rightarrow \Omega_{\varphi(P)}$. If $\varphi(0)=0$ then $\Phi_{\varphi}\left(\Xi_{P}\right)=\Xi_{\varphi(P)}$. If $\varphi$ is surjective then $\Phi_{\varphi}$ is surjective.

Proof. Choose $r>0$ and then $r^{\prime} \geq r$ according to Lemma 4.3. Let $D(P-x, P-y)<\frac{1}{r^{\prime}}$. Since $P$ has finite local complexity we can find $x^{\prime}, y^{\prime} \in B\left(0, \frac{1}{2 r^{\prime}}\right)$ such that $B_{r^{\prime}}\left[P-x-x^{\prime}\right]=B_{r^{\prime}}\left[P-y-y^{\prime}\right]$. By Lemma 4.3 we then have $B_{r}\left[\varphi(P)-\varphi\left(x+x^{\prime}\right)\right]=B_{r}\left[\varphi(P)-\varphi\left(y+y^{\prime}\right)\right]$ and so $D\left(\varphi(P)-\varphi\left(x+x^{\prime}\right), \varphi(P)-\varphi\left(y+y^{\prime}\right)\right)<\frac{1}{r}$. Since $P$ has finite local complexity $d \varphi$ is bounded and $\left|\varphi\left(x+x^{\prime}\right)-\varphi(x)\right| \leq\|d \varphi\|_{\infty}\left|x^{\prime}\right|$. If $r$ is sufficiently large then $D\left(\varphi(P)-\varphi\left(x+x^{\prime}\right), \varphi(P)-\varphi(x)\right) \leq\|d \varphi\|_{\infty}\left|x^{\prime}\right|$. Hence the triangle inequality gives

$$
D(\varphi(P)-\varphi(x), \varphi(P)-\varphi(y)) \leq \frac{1}{r}+\frac{\|d \varphi\|_{\infty}}{2 r^{\prime}}+\frac{\|d \varphi\|_{\infty}}{2 r^{\prime}} .
$$

This tending to 0 for $r$ tending to infinity we conclude that $\Phi_{\varphi}$ is uniformly continuous.

That $\Phi_{\varphi}$ preserves the canonical transversal provided $\varphi(0)=0$ follows immediately from the construction of $\Phi_{\varphi}$. Since $\Phi_{\varphi}\left(\Xi_{P}\right)$ is closed and contains $\{\varphi(P)-\varphi(p) \mid p \in P\}$ we must have $\Phi_{\varphi}\left(\Xi_{P}\right)=\Xi_{\varphi(P)}$.

Finally, if $\varphi$ is surjective then $\operatorname{orb}\left(P^{\prime}\right)=\Phi_{\varphi}(\operatorname{orb}(P))$ which is dense and hence implies surjectivity of $\Phi_{\varphi}$. 
Note that since $d \varphi$ is strongly $P$-equivariant $\varphi(P)$ has finite local complexity, but it need not be a Delone set.

We are now interested in the converse direction: given a continuous map between hulls of Delone sets, can we associate a strongly $P$-equivariant 1 -form to it?

Let $P, P^{\prime}$ be aperiodic Delone sets of finite local complexity and $\Phi: \Omega_{P} \rightarrow \Omega_{P^{\prime}}$ a continuous map satisfying $\Phi(P)=P^{\prime}$. Since the orbits under the translation action are the path-connected components $\Phi$ must preserve orbits. Hence for any $x$ exists a $x^{\prime}$ such that $\Phi(P-x)=$ $P^{\prime}-x^{\prime}$. Since $P^{\prime}$ is aperiodic $x^{\prime}$ is uniquely determined by $x$. Thus there exists a family of functions $\varphi_{P-y}: \mathbb{R}^{n} \rightarrow \mathbb{R}^{n}, y \in \mathbb{R}^{n}$ such that

$$
\Phi(P-y-x)=P^{\prime}-y-\varphi_{P-y}(x), \quad \varphi_{P-y}(0)=0 .
$$

Lemma 4.9. For all $y \in \mathbb{R}^{n}, \varphi_{P-y}: \mathbb{R}^{n} \rightarrow \mathbb{R}^{n}$ is uniformly continuous. Furthermore, for all $x \in \mathbb{R}^{n}$, orb $(P) \ni \omega \mapsto \varphi_{\omega}(x) \in \mathbb{R}^{n}$ is uniformly continuous.

Proof. To show that $\varphi_{P}$ is uniformly continuous choose $0<\epsilon<\frac{r_{\min }\left(P^{\prime}\right)}{3}$, where $r_{\text {min }}\left(P^{\prime}\right)$ is the minimal distance in $P^{\prime}$. By the uniform continuity of $\Phi$ there exists $\delta$ such that for all $x_{0} \in \mathbb{R}^{n},|v|<\delta$ implies

$$
D\left(\Phi\left(P-x_{0}\right), \Phi\left(P-x_{0}-v\right)\right)<\epsilon .
$$

By the remarks at the end of Sect. 2.1 the pre-image under $w \mapsto \Phi(P-$ $\left.x_{0}\right)-w$ of the path-connected component of $\Phi\left(P-x_{0}\right)$ in $U_{\epsilon}\left(\Phi\left(P-x_{0}\right)\right)$ is contained in $B(0, \epsilon)$. Therefore $\varphi_{P-x_{0}}(B(0, \delta)) \subset B\left(0, \frac{r_{\min }\left(P^{\prime}\right)}{3}\right)$.

Furthermore, (4.5) implies that either $\left|\varphi_{P}\left(x_{0}+v\right)-\varphi_{P}\left(x_{0}\right)\right| \leq \epsilon$ or $\left|\varphi_{P}\left(x_{0}+v\right)-\varphi_{P}\left(x_{0}\right)\right| \geq r_{m i n}\left(P^{\prime}\right)-\epsilon$. But the second possibility cannot hold since $\left|\varphi_{P}\left(x_{0}+v\right)-\varphi_{P}\left(x_{0}\right)\right|=\left|\varphi_{P-x_{0}}(v)\right| \leq \frac{r_{\min }\left(P^{\prime}\right)}{3}$. This proves uniform continuity of $\varphi_{P}$. That of $\varphi_{P-y}$ follows in the same way.

To show that $\omega \mapsto \varphi_{\omega}(y)$ is uniformly continuous on the orbit of $P$ we choose $\epsilon>0$, small compared with $|y|^{-1}$. Note that $B_{r}[\omega]=B_{r}\left[\omega^{\prime}\right]$ implies $B_{r-|x|}[\omega-x]=B_{r-|x|}\left[\omega^{\prime}-x\right]$ provided $r>|x|$. Using this property, the (uniform) continuity of $\Phi$ and boundedness of $\varphi_{\omega^{\prime}}$ we can find $\delta>0$ such that $D\left(\omega, \omega^{\prime}\right)<\delta$ implies for all $y^{\prime} \in B(0,|y|)$ : $D\left(\Phi\left(\omega-y^{\prime}\right), \Phi\left(\omega^{\prime}-y^{\prime}\right)\right)<\epsilon$ and $D\left(\Phi(\omega)-\varphi_{\omega^{\prime}}\left(y^{\prime}\right), \Phi\left(\omega^{\prime}\right)-\varphi_{\omega^{\prime}}\left(y^{\prime}\right)\right)<\epsilon$ and therefore

$$
D\left(\Phi(\omega)-\varphi_{\omega^{\prime}}\left(y^{\prime}\right), \Phi(\omega)-\varphi_{\omega}\left(y^{\prime}\right)\right)<2 \epsilon .
$$

This implies that $\left|\varphi_{\omega}\left(y^{\prime}\right)-\varphi_{\omega^{\prime}}\left(y^{\prime}\right)\right|$ is either smaller than $2 \epsilon$ or larger than $r_{\min }\left(P^{\prime}\right)-2 \epsilon$. From the continuity of $\varphi_{\omega}$ and the condition $\varphi_{\omega}(0)=$ 0 follows therefore that $\left|\varphi_{\omega}(y)-\varphi_{\omega^{\prime}}(y)\right|<2 \epsilon$ (for small enough $\epsilon$ ). 
We now require in addition that $\Phi$ preserves the canonical transversal.

Theorem 4.10. Let $P, P^{\prime}$ be aperiodic Delone sets of finite local complexity. Let $\Phi: \Omega_{P} \rightarrow \Omega_{P^{\prime}}$ be a continuous map satisfying $\Phi\left(\Xi_{P}\right) \subset \Xi_{P^{\prime}}$ and $\Phi(P)=P^{\prime}$. Then there exists a smooth function $\varphi: \mathbb{R}^{n} \rightarrow \mathbb{R}^{n}$ with strongly $P$-equivariant differential which coincides with $\varphi_{P}$ (as defined by (4.4)) on $P$ and satisfies $\varphi(P) \subset P^{\prime}$.

Proof. We can sharpen the last part of the proof of Lemma 4.9 provided $w, w^{\prime} \in \Xi_{P}$ and $y=P-P$. Let $\epsilon>0, M>0$ and $p, q \in P$. By the reasoning of the proof of Lemma 4.9 there exists $R>M$ such that $B_{R}[P-p]=B_{R}[P-q]$ implies for all $y \in B_{M}[P-p]: D(\Phi(P-p-$ $y), \Phi(P-q-y))<\epsilon$ and $\left|\varphi_{P-p}(y)-\varphi_{P-q}(y)\right|<\epsilon$. Moreover, $\Phi(P-p-$ $y)$ and $\Phi(P-q-y)$ lie in $\Xi_{P^{\prime}}$ and hence the finite local complexity of $P^{\prime}$ allows us to replace $D$ by $D_{0}$ to obtain $B_{\frac{1}{c}}[\Phi(P-p-y)]=B_{\frac{1}{\epsilon}}[\Phi(P-$ $q-y)]$. Hence $\left|\varphi_{P-p}(y)-\varphi_{P-q}(y)\right|<\epsilon$ forces $\left|\varphi_{P-p}(y)-\varphi_{P-q}(y)\right|$ to vanish. A simple calculation shows that $\varphi_{P-p}(y)=\varphi_{P}(p+y)-\varphi_{P}(p)$. Hence for all $h \in P-P, \Delta_{h} \varphi_{P}: P \cap(P-h) \rightarrow \mathbb{R}^{n}$ is strongly $P$ equivariant. Applying Lemma [2.6, we obtain a smooth function $\varphi$ with strongly $P$-equivariant differential which coincides with $\varphi_{P}$ on $P$.

Finally, if $p \in P$ then $\Phi(P-p) \in \Xi_{P^{\prime}}$ and hence $0 \in P^{\prime}-\varphi(p)$. This shows that $\varphi(P) \subset P^{\prime}$.

We cannot expect that under the hypothesis of the theorem $\varphi(P)$ is all of $P^{\prime}$. For instance, if $P^{\prime}$ is obtained from $P$ by adding points in a locally derivable way then $\varphi=$ id would be the resulting map but $P$ a proper subset of $P^{\prime}$.

Corollary 4.11. Let $P, P^{\prime}$ be aperiodic Delone sets of finite local complexity. Let $\Phi: \Omega_{P} \rightarrow \Omega_{P^{\prime}}$ be a homeomorphism which maps $\Xi_{P}$ onto $\Xi_{P^{\prime}}$ and $P$ to $P^{\prime}$. Then the map $\varphi$ constructed in the theorem satisfies Hypothesis 4.1 and $P^{\prime}=\varphi(P)$.

Proof. Since $\Phi$ is a homeomorphism also $\varphi_{P}$ is a homeomorphism and its inverse $\varphi_{P}^{-1}$ is the map constructed in (4.4) from $\Phi^{-1}: \Omega_{P^{\prime}} \rightarrow \Omega_{P}$, we denote it here by $\varphi_{P^{\prime}}$. By Lemma 4.9 it is uniformly continuous and hence we can find at least one $r>0$ for which $\exists r^{\prime}>0, \forall y \in \mathbb{R}^{n}$ : $\varphi_{P^{\prime}}(B(y, r)) \subset B\left(\varphi_{P^{\prime}}(y), r^{\prime}\right)$. Our aim is to show that this implies that $\forall r>0, \exists r^{\prime}>0, \forall y \in \mathbb{R}^{n}: \varphi_{P^{\prime}}(B(y, r)) \subset B\left(\varphi_{P^{\prime}}(y), r^{\prime}\right)$ and hence that $\varphi_{P}$ satisfies (4.1). To see this let $0 \in A \subset B(0, r)$ be a finite set such that $B\left(0, \frac{3}{2} r\right) \subset \bigcup_{a \in A} B(a, r)$. Then the above implies

$$
\varphi_{P^{\prime}}\left(B\left(y, \frac{3}{2} r\right)\right) \subset \bigcup_{a \in A} B\left(\varphi_{P^{\prime}}(y+a), r^{\prime}\right) .
$$


By Lemma 4.9 the maps $y \mapsto \varphi_{P^{\prime}}(y+a)-\varphi_{P^{\prime}}(y)$ are weakly $P^{\prime}$ equivariant and hence bounded. If we denote a common bound for their norm by $M$ then $\bigcup_{a \in A} B\left(\varphi_{P^{\prime}}(y+a), r^{\prime}\right) \subset B\left(\varphi_{P^{\prime}}(y), r^{\prime}+M\right)$. This shows that $\varphi_{P}$ satisfies (4.1). Since $\varphi_{P}$ and $\varphi$ are uniformly continuous ( $\varphi$ as $d \varphi$ is bounded), $\varphi_{P}-\varphi$ is bounded and hence $\varphi$ satisfies Hypothesis 4.1 .

We have $\Phi(\operatorname{orb}(P))=\operatorname{orb}\left(P^{\prime}\right)$ and $\Phi^{-1}\left(\Xi_{P^{\prime}}\right)=\Xi_{P}$. Now if $y \in P^{\prime}$ then the first identity implies that there exists a $x$ such that $P^{\prime}-$ $\varphi_{P}(x)=P^{\prime}-y$, and hence $\varphi_{P}(x)-y$ lies in the periodicity lattice of $P^{\prime}$. Using $\varphi(0)=0$ and continuity we can arrange for $\varphi_{P}(x)=y$. Now the second identity implies that $P-x \in \Xi_{P}$ and hence $x \in P$.

Let us call two homeomorphisms from the hull of $P$ into the hull of another pattern $P^{\prime}$ which preserve the canonical transversals and map $P$ to $P^{\prime}$ equivalent if the maps constructed in (4.4) for $y=0$ coincide on $P$. Then we may say that a small neighbourhood of the class of did in $Z_{s-P}^{1}\left(\mathbb{R}^{n}, \mathbb{R}^{n}\right) / N_{s-P}^{1}\left(\mathbb{R}^{n}, \mathbb{R}^{n}\right)$ parametrises equivalence classes of such homeomorphisms.

Remark 4.12. The map $\varphi$ constructed in Theorem 4.10 may differ substantially from $\varphi_{P}$ away from $P$. A refinement of Lemma 2.6.3 allows us to construct maps which are arbitrarily close, namely for all $\epsilon>0$ there exists a smooth function $\varphi: \mathbb{R}^{n} \rightarrow \mathbb{R}^{n}$ with strongly $P$-equivariant differential such that $\left\|\varphi_{P}-\varphi\right\|_{\infty}<\epsilon$ and $\varphi$ coincides with $\varphi_{P}$ on $P$.

This can be seen as follows. Fix $\delta>0$. Uniform continuity of $\omega \mapsto$ $\varphi_{\omega}(x)$ is equivalent to weak $P$-equivariance of $y \mapsto \varphi_{P-y}(x)=\Delta_{x} \varphi_{P}(y)$ $\left(x \in \mathbb{R}^{n}\right.$ fixed) 2 Hence we can find a partition $\left\{P_{i}\right\}_{i}$ of $P$ such that for all $x \in B(0,3 A)$ and $p, q \in P_{i}$ we have $\left|\Delta_{x} \varphi_{P}(p)-\Delta_{x} \varphi_{P}(q)\right|<\delta$. Furthermore, taking if neccessary a refinement of that partition the strong $P$-equivariance of $\Delta_{h} \varphi_{P}: P \cap(P-h) \rightarrow \mathbb{R}^{n}$ allows us to assume that for $h \in P-P,|h| \leq A, \Delta_{h} \varphi_{P}$ takes the same value on all points of $P_{i}$. Choose $p_{i} \in P_{i}$ and set, for $p \in P_{i}$ and $x \in \operatorname{int} V_{p}-p$,

$$
\tilde{\phi}(x+p)=\psi\left(x+p_{i}\right)-\psi\left(p_{i}\right)+\psi(p) .
$$

Now we proceed exactly as in the proof of Lemma 2.613 thereby obtaining a smooth function $\varphi=\rho * \tilde{\phi}$ which has strongly $P$-equivariant differential. If we choose $\delta$ small enough then $\left\|\varphi-\varphi_{P}\right\|_{\infty}$ is smaller than any given $\epsilon$. The only drawback is that $\varphi-\varphi_{P}$ does not vanish on $P$. But the restriction of $\varphi-\varphi_{P}$ to $P$ is strongly $P$-equivariant. We may therefore use Lemma 2.6.3 to add a smooth strongly $P$-equivariant function to $\varphi$ such that the result coincides with $\varphi_{P}$ on $P$. This correction is of order $\epsilon$ in the $\|\cdot\|_{\infty}$-norm and so we are done.

\footnotetext{
${ }^{2}$ We extend the definition of $\Delta_{x} \varphi_{P}(y)=\varphi_{P}(y+x)-\varphi_{P}(y)$ to $x, y \in \mathbb{R}^{n}$.
} 
4.2. Exact $P$-equivariant 1 -forms. Exact $P$-equivariant 1 -forms are of the form $d f$ where $f$ is $P$-equivariant. This implies that $f$ is bounded. Such maps are not good candidates to compare $P$ with $f(P)$ as the latter could not even be uniformly discrete. It is more fruitful to consider exact one forms of the form $d(\varphi-\mathrm{id})$ and then compare $P$ with $\varphi(P)$.

Theorem 4.13. Let $P$ be a Delone set of finite local complexity, $\varphi$ : $\mathbb{R}^{n} \rightarrow \mathbb{R}^{n}$ be a $C^{0}$-function and $g \in G L(n, \mathbb{R})$. Suppose that $\varphi-g$ is weakly P-equivariant. Then $\Phi_{g}$ is a uniformly continuous map which satisfies $\Phi_{g}(\omega-x)=\Phi_{g}(\omega)-g(x)$. In particular $\Phi_{\mathrm{id}}$ extends to a topological semi-conjugacy between $\left(\Omega_{P}, \mathbb{R}^{n}\right)$ and $\left(\Omega_{\varphi(P)}, \mathbb{R}^{n}\right)$ mapping $P$ to $P^{\prime}$. If $\varphi$ is even strongly $P$-equivariant then $\varphi(P)$ is a Delone set.

Proof. We consider first the case that $\varphi-g$ is $P$-equivariant with range $R$. Clearly $g(P)$ is a Delone set. Since $\varphi(P)=g(P)+(\varphi-g)(P)$ and $(\varphi-g)(P)$ is finite by Lemma 2.4, also $\varphi(P)$ is a Delone set. For the second statement choose $r>0$ and let $r^{\prime}=R+r$. Let $D(P-$ $x, P-y)<\frac{1}{r^{\prime}}$. Since $P$ has finite local complexity we can find $x^{\prime}, y^{\prime} \in$ $B\left(0, \frac{1}{2 r^{\prime}}\right)$ such that $B_{r^{\prime}}\left[P-x-x^{\prime}\right]=B_{r^{\prime}}\left[P-y-y^{\prime}\right]$. By Lemma 4.2 we then have $B_{r}\left[\varphi(P)-g\left(x+x^{\prime}\right)\right]=B_{r}\left[\varphi(P)-g\left(y+y^{\prime}\right)\right]$ and so $D\left(\varphi(P)-g\left(x+x^{\prime}\right), \varphi(P)-g\left(y+y^{\prime}\right)\right)<\frac{1}{r}$. If $r$ is sufficiently large then $D\left(\varphi(P)-g\left(x+x^{\prime}\right), \varphi(P)-g(x)\right) \leq\left|g\left(x^{\prime}\right)\right| \leq \frac{\|g\|_{\infty}}{2 r^{\prime}}$. Hence the triangle inequality gives

$$
D(P-g(x), P-g(y)) \leq \frac{1}{r}+\frac{\|g\|_{\infty}}{R+r} .
$$

Since this tends to 0 when $r$ tends to infinity we conclude that $\Phi_{g}$ is uniformly continuous.

Now suppose that $\varphi-g$ is merely weakly $P$-equivariant and hence for any $\epsilon>0$ exists a strongly $P$-equivariant $C^{0}$-function $\varphi_{\epsilon}$ such that $\left\|\varphi-\varphi_{\epsilon}\right\|_{\infty}<\epsilon$. Now

$$
D\left(\Phi_{g}(P-x), \Phi_{\epsilon g}(P-x)\right) \leq d_{H}\left(\varphi(P), \varphi_{\epsilon}(P)\right) \leq \epsilon
$$

and a further application of the triangle inequality implies that $\Phi_{g}$ is uniformly continuous.

The surjectivity of $g$ implies that the image of $\Phi_{g}$ contains the orbit of $\varphi(P)$ and hence that $\Phi_{g}$ is surjective. The relation $\Phi_{g}(\omega-x)=$ $\Phi_{g}(\omega)-g(x)$ is clear for $\omega=P-y$ and follows for general $\omega$ from the continuity of $\Phi_{g}$.

We now investigate conditions which lead to statements in the converse direction: Assuming that $P^{\prime}$ is a deformation of $P$ such that the dynamical systems are pointed topological conjugate, is there a weakly 
$P$-equivariant map $\varphi$ such that $\varphi(P)=P^{\prime}$ ? Clark and Sadun obtained related results for substitution tilings CS06.

Consider two patterns $P$ and $P^{\prime}$ of finite local complexity and suppose that $\Psi: \Omega_{P} \rightarrow \Omega_{P^{\prime}}$ is a semi-conjugacy mapping $P$ to $P^{\prime}$. Since it commutes with the $\mathbb{R}^{n}$ actions the maps constructed in Lemma 4.9 are all equal to the identity. Moreover, since $\Psi$ has to be the continuous extension of $P-x \mapsto P^{\prime}-x$ we must have

$$
\Psi=\Phi_{\text {id }}
$$

Of course, the semi-conjugacy is not assumed to preserve the canonical transversals. If we assume in addition that $P^{\prime}$ is the image of $P$ under a $C^{1}$-function $\varphi$ satisfying Hypothesis 4.1 and having strongly $P$-equivariant differential we obtain from Theorem 4.8 a (in general different) map $\Phi_{\varphi}: \Omega_{P} \rightarrow \Omega_{P^{\prime}}$ which preserves the canonical transversal but does not commute with the $\mathbb{R}^{n}$ actions. Our main question is therefore, under which additional conditions does uniform continuity of $\Phi_{\mathrm{id}}$ and $\Phi_{\varphi}$ imply that $\varphi-$ id is weakly $P$-equivariant?

A first observation to make is that boundedness of $\varphi-\mathrm{id}$ is neccessary, as weakly $P$-equivariant functions are bounded. We show below that boundedness of $\varphi-$ id is sufficient, provided $P^{\prime}$ is aperiodic and uniformly continuous.

Lemma 4.14. Let $P \subset \mathbb{R}^{n}$ be an aperiodic, uniformly discrete set. For all $M>0$ there exists $r>0$ such that for all $h \in \mathbb{R}^{n},|h| \leq M$ holds: if $B_{r}[P]=B_{r}[P-h]$ then $h=0$.

Proof. Let us suppose the contrary. Then there exist $M>0$, a sequence $\left(r_{n}\right)_{n}$ tending to $+\infty$, and points $h_{n} \in \mathbb{R}^{n},\left|h_{n}\right| \leq M$ such that $B_{r_{n}}[P]=$ $B_{r_{n}}\left[P-h_{n}\right]$ but $h_{n} \neq 0$. Since $\left\{h \in \mathbb{R}^{n}:|h| \leq M\right\}$ is compact we can assume, perhaps after taking a sub-sequence, that $\left(h_{n}\right)_{n}$ converges, let's say to $h$. This limit satisfies $h \neq 0$ since $B_{r_{n}}[P]=B_{r_{n}}\left[P-h_{n}\right]$ for large enough $r_{n}$ implies that either $h_{n}=0$ or $\left|h_{n}\right| \geq r_{\min }(P)$. It follows that $\left(P-h_{n}\right)_{n}$ converges to $P-h$ and that $P-h=P$. This contradicts the assumption that $P$ is aperiodic.

Theorem 4.15. Let $P^{\prime}$ be an aperiodic and uniformly discrete deformation of a Delone set $P$, i.e. in particular $P^{\prime}=\varphi(P)$ for a smooth function $\varphi: \mathbb{R}^{n} \rightarrow \mathbb{R}^{n}$ with strongly $P$-equivariant differential and satisfying Hypothesis 4.1. Suppose that $P^{\prime}$ is moreover pointed topologically semi-conjugate to $P$ and hence $\Phi_{\mathrm{id}}$ is uniformly continuous. If $\varphi-\mathrm{id}$ is bounded then it is weakly P-equivariant.

Proof. Without loss of generality we may assume that $\varphi(0)=0$. Then $\Phi_{\varphi}: \Omega_{P} \rightarrow \Omega_{P^{\prime}}$, which is uniformly continuous by Theorem 4.8, is 
given by

$$
\Phi_{\varphi}(P-x)=\Phi_{\mathrm{id}}(P-x)+\eta(x)
$$

where $\eta=\varphi-$ id. By definition of the metric, uniform continuity of $\Phi_{\varphi}$ and $\Phi_{\text {id }}$ imply that for any $\epsilon>0$ exists $\delta>0$ such that for all $x, y \in \mathbb{R}^{n}$ :

$$
\begin{aligned}
& B_{\delta^{-1}}[P-x]=B_{\delta^{-1}}[P-y] \\
& \Longrightarrow \quad\left\{\begin{array}{l}
B_{\frac{1}{\epsilon}}\left[\Phi_{\varphi}(P-x)\right] \cong_{\epsilon} B_{\frac{1}{\epsilon}}\left[\Phi_{\varphi}(P-y)\right] \\
B_{\frac{1}{\epsilon}}\left[\Phi_{\varphi}(P-x)+\eta(x)\right] \cong_{\epsilon} B_{\frac{1}{\epsilon}}\left[\Phi_{\varphi}(P-y)+\eta(y)\right]
\end{array}\right.
\end{aligned}
$$

Suppose $\epsilon^{-1}>2\left(\|\eta\|_{\infty}+1\right)$. Let $p \in P$. Then we have for all $x \in \mathbb{R}^{n}$ :

$$
\begin{aligned}
& B_{\delta^{-1}}[P-x]=B_{\delta^{-1}}[P-p] \Longrightarrow \exists x^{\prime} \in \mathbb{R}^{n},\left|x^{\prime}\right|<2 \epsilon: \\
& \quad B_{\frac{1}{2} \epsilon^{-1}}\left[\Phi_{\varphi}(P-p)\right]=B_{\frac{1}{2} \epsilon^{-1}}\left[\Phi_{\varphi}(P-p)+\eta(x)-\eta(p)-x^{\prime}\right] .
\end{aligned}
$$

Since $\eta$ is bounded we can apply Lemma 4.14 to $\Phi_{\varphi}(P-p)$ with $h=$ $\eta(x)-\eta(p)-x^{\prime}$ to conclude that $|\eta(x)-\eta(p)|<2 \epsilon$. By the finite local complexity of $P$ there exists a finite subset $\left\{p_{1}, \cdots, p_{k}\right\} \subset P$ such that the sets $\left\{p \in P \mid B_{\delta^{-1}}[P-p]=B_{\delta^{-1}}\left[P-p_{i}\right]\right\}$ partition $P$. They are clearly locally derivable from $P$. Hence we can apply Prop. 2.7 to obtain the statement.

The following example shows that the hypothesis of boundedness of $\varphi-$ id is not implied by the other conditions of the theorem. The Penrose tilings can be defined by means of a substitution. This is a locally defined decomposition $\gamma$ of the tiles into smaller tiles followed by a rescaling by factor of $\tau$, the golden ratio. If $T$ is a Penrose tiling then $\gamma(T)$ and $T$ are mutually locally derivable, since the substitution is recognisable. In particular, $T$ and $\gamma(T)$ are pointed topologically conjugate and so are their vertex sets $T^{v}$ and $\gamma(T)^{v}$. Now a few Penrose tilings have the property that they are invariant under the forth iterate of the substitution. Hence for those, $\gamma^{4}(T)^{v}=\tau^{-4} T^{v}$, and thus $\gamma^{4}(T)^{v}$ is a deformation of $T^{v}$ given by the map $\varphi=\tau^{-4}$ id. Clearly $\varphi-$ id is unbounded.

4.3. Deforming back. $\varphi(P)$ being a deformation of $P$ does not imply that $P$ is a deformation of $\varphi(P)$, i.e. deformations are not always invertible (for instance if $\varphi(P)$ is periodic whereas $P$ was not). Although this is not analysed in [CS06], it is quite clear that, for fixed $k$ (cf. Section 2.4.2), there exists a neighbourhood of the identity shape function such that shape functions in that neighbourhood are admissible and define deformations which can be inverted. It is not clear apriori however, that one can obtain a lower bound on the size of this neighbourhood which is uniform in $k$. We will in this section effectively 
establish such a lower bound (Theorem 4.18) and thereby guarantee that small deformations are invertible, i.e. can be deformed back.

Given a uniformly discrete set $P$ with minimal distance between points $r_{\text {min }}$ we define $A_{P}:\left[r_{\min }, \infty\right) \rightarrow \mathbb{R}^{+}$,

$$
A_{P}(r)=\inf \left\{\left|h-h^{\prime}\right|: h, h^{\prime} \in \bigcup_{x \in P} B_{r}[P-x], h \neq h^{\prime}\right\} .
$$

$A_{P}(r)$ is a monotonically decreasing function which is bounded from above by $r_{\text {min }}$. Moreover, if $P$ has finite local complexity then $A_{P}(r)$ is strictly positive since then $\bigcup_{x \in P} B_{r}[P-x]$ is a finite set.

For the following lemma and its corollary we do not require any $P$ equivariance property of $\varphi$ or $d \varphi$. In particular, $\varphi(P)$ will usually not have finite local complexity.

Lemma 4.16. Let $P$ be a uniformly discrete set of finite local complexity. For all $r>0$ exists $1>\epsilon>0$ such that for all differentiable functions $\varphi: \mathbb{R}^{n} \rightarrow \mathbb{R}^{n}$ which satisfy $\|d(\varphi-\mathrm{id})\|_{\infty}<\epsilon$ holds: $\forall x, y \in P$ :

$$
\begin{aligned}
& B_{r^{\prime}}[\varphi(P)-\varphi(x)]=B_{r^{\prime}}[\varphi(P)-\varphi(y)] \Longrightarrow \\
& \quad\left\{\begin{array}{l}
B_{r}[P-x]=B_{r}[P-y] \text { and } \\
\forall h \in B_{r}[P-x]: \varphi(x+h)-\varphi(x)=\varphi(y+h)-\varphi(y)
\end{array}\right.
\end{aligned}
$$

where $r^{\prime}=r(1-\epsilon)^{-1}$.

Proof. The statement is trivial for $r<r_{\text {min }}$, the minimal distance in $P$, so we assume $r \geq r_{\min }$. Define

$$
\epsilon=\sup _{0<t<1}\left\{2 t(1-t)^{-2} r \leq A_{P}\left((1-t)^{-2} r\right)\right\} .
$$

Suppose that $\|d(\varphi-\mathrm{id})\|_{\infty}<\epsilon$. Let $r^{\prime}=r(1-\epsilon)^{-1}$ and $S_{r^{\prime}}(x):=\{h \in$ $\left.P-x \mid \varphi(x+h)-\varphi(x) \in B_{r^{\prime}}[\varphi(P)-\varphi(x)]\right\}$. Since $\varphi$ is bi-Lipschitz with constant $\lambda=(1-\epsilon)^{-1}$ we have

$$
B_{(1-\epsilon) r^{\prime}}[P-x] \subset S_{r^{\prime}}(x) \subset B_{(1-\epsilon)^{-1} r^{\prime}}[P-x] .
$$

Now let $B_{r^{\prime}}[\varphi(P)-\varphi(x)]=B_{r^{\prime}}[\varphi(P)-\varphi(y)]$. This implies that there exists a bijection $\beta: S_{r^{\prime}}(x) \rightarrow S_{r^{\prime}}(y)$ such that

$$
\varphi(y+\beta(h))-\varphi(y)=\varphi(x+h)-\varphi(x) .
$$

It follows that $\beta(h)-h=\eta(x+h)-\eta(x))-\eta(y+\beta(h))-\eta(y)$ with $\eta=\varphi-\mathrm{id}$ and hence

$$
|\beta(h)-h| \leq(|h|+|\beta(h)|)\|d(\varphi-\mathrm{id})\|_{\infty} .
$$

By the right inclusion of (4.7)

$$
(|h|+|\beta(h)|)\|d(\varphi-\mathrm{id})\|_{\infty}<2(1-\epsilon)^{-1} r^{\prime} \epsilon=A_{P}\left((1-\epsilon)^{-1} r^{\prime}\right) .
$$


Since $\beta(h), h \in \bigcup_{x \in P} B_{r}[P-x]$ the definition of $A_{P}$ implies that $h=$ $\beta(h)$ and so $S_{r^{\prime}}(x)=S_{r^{\prime}}(y)$. From the left inclusion of (4.7) follows that $B_{r}[P-x]=B_{r}[P-y]$ and, since $h=\beta(h), \forall h \in B_{r}[P-x]$ : $\varphi(x+h)-\varphi(x)=\varphi(y+h)-\varphi(y)$.

Corollary 4.17. Let $P$ be a Delone set of finite local complexity. There exists $1>\epsilon>0$ such that for all differentiable functions $\varphi: \mathbb{R}^{n} \rightarrow \mathbb{R}^{n}$ which satisfy $\|d(\varphi-\mathrm{id})\|_{\infty}<\epsilon$ holds: $\forall r>0 \exists r^{\prime}>0 \forall x, y \in P$ :

$$
B_{r^{\prime}}[\varphi(P)-\varphi(x)]=B_{r^{\prime}}[\varphi(P)-\varphi(y)] \Longrightarrow B_{r}[P-x]=B_{r}[P-y]
$$

Proof. Choose $\delta>0$ and $r_{0}>0$ such that for all $r \geq r_{0}$ and $x \in P$ holds

$$
B_{r+\delta}[P-x] \subset \bigcup_{h \in B_{r}[P-x]} B_{r}[P-x-h]+h .
$$

This is possible since $P$ is relatively dense. Choose $\epsilon$ such that the statement of Lemma 4.16 is satisfied with $r=r_{0}$ and some $r^{\prime}=r_{0}^{\prime}$. Let, for $x \in P$,

$$
V(x):=\bigcup_{h \in B_{r}[P-x]} B_{r_{0}^{\prime}}[\varphi(P)-\varphi(x+h)]+\varphi(x+h)-\varphi(x) .
$$

$V(x)$ is a subset of $\varphi(P)-\varphi(x)$. Then

$$
B\left(\varphi(x+h)-\varphi(x), r_{0}^{\prime}\right) \cap V(x)=B_{r_{0}^{\prime}}[\varphi(P)-\varphi(x+h)]+\varphi(x+h)-\varphi(x) .
$$

Now let $V(x)=V(y), x, y \in P$. This implies in particular $B_{r_{0}^{\prime}}[\varphi(P)-$ $\varphi(x)]=B_{r_{0}^{\prime}}[\varphi(P)-\varphi(y)]$ from which we conclude from Lemma 4.16 that $B_{r_{0}}[P-x]=B_{r_{0}}[P-y]$ and $\forall h \in B_{r_{0}}[P-x]: \varphi(x+h)-\varphi(x)=$ $\varphi(y+h)-\varphi(y)$. The latter implies with (4.10) that $\forall h \in B_{r_{0}}[P-x]$ : $B_{r_{0}^{\prime}}[\varphi(P)-\varphi(x+h)]=B_{r_{0}^{\prime}}[\varphi(P)-\varphi(y+h)]$ and hence by Lemma 4.16 that $\forall h \in B_{r_{0}}[P-x]$ holds $B_{r_{0}}[P-x-h]=B_{r_{0}}[P-y-h]$ and $\forall h^{\prime} \in B_{r_{0}}[P-x-h]: \varphi\left(x+h+h^{\prime}\right)-\varphi(x+h)=\varphi\left(y+h+h^{\prime}\right)-\varphi(y+h)$. In particular we find $\forall h^{\prime \prime} \in B_{r_{0}}[P-x-h]+h$ :

$$
\begin{aligned}
\varphi\left(x+h^{\prime \prime}\right)-\varphi(x) & =\varphi\left(x+h+\left(h^{\prime \prime}-h\right)\right)-\varphi(x+h)+\varphi(x+h)-\varphi(x) \\
& =\varphi\left(y+h+\left(h^{\prime \prime}-h\right)\right)-\varphi(y+h)+\varphi(y+h)-\varphi(y) \\
& =\varphi\left(y+h^{\prime \prime}\right)-\varphi(y),
\end{aligned}
$$

as $h^{\prime \prime}-h \in B_{r_{0}}[P-x-h]$. Thus $V(x)=V(y), x, y \in P$ implies $B_{r_{1}}[P-x]=B_{r_{1}}[P-y]$ and $\forall h \in B_{r_{1}}[P-x]: \varphi(x+h)-\varphi(x)=$ $\varphi(y+h)-\varphi(y)$ where $r_{1}=r_{0}+\delta$. Since $V(x)$ is finite there exists $r_{1}^{\prime}$ such that $V(x) \subset B\left(0, r_{1}^{\prime}\right)$. It follows that, for $x, y \in P, B_{r_{1}^{\prime}}[\varphi(P)-\varphi(x)]=$ $B_{r_{1}^{\prime}}[\varphi(P)-\varphi(y)]$ implies $V(x)=V(y)$. We have thus proven that the 
statement of Lemma 4.16 holds with the same $\epsilon$ but $r=r_{1}$ and $r^{\prime}=r_{1}^{\prime}$. Iterating this argument yields the statement of the corollary.

Note that in the statement of the corollary the points $x$ and $y$ were taken from $P$ and so the corollary does not imply that $d \varphi^{-1}$ is strongly $P$-equivariant 3 To obtain the statement for arbitrary $x, y \in \mathbb{R}^{n}$ we need to require that $d \varphi$ is strongly $P$-equivariant.

Theorem 4.18. Let $P$ be a Delone set of finite local complexity. There exists $1>\epsilon>0$ such that for all differentiable functions $\varphi: \mathbb{R}^{n} \rightarrow$ $\mathbb{R}^{n}$ whose differential satisfies $\|d(\varphi-\mathrm{id})\|_{\infty}<\epsilon$ and is strongly $P$ equivariant holds: $\forall r>0 \exists r^{\prime}>0 \forall x, y \in \mathbb{R}^{n}$

$$
B_{r^{\prime}}[\varphi(P)-\varphi(x)]=B_{r^{\prime}}[\varphi(P)-\varphi(y)] \Longrightarrow B_{r}[P-x]=B_{r}[P-y] \text {. }
$$

Proof. Given $P$ and $\epsilon$ according to Corollary 4.17 let $\varphi: \mathbb{R}^{n} \rightarrow \mathbb{R}^{n}$ be a $C^{1}$-function whose differential $d \varphi$ is $P$-equivariant with range $R$ and satisfies $\|d(\varphi-\mathrm{id})\|_{\infty}<\epsilon$. Let $r \geq \max \left\{\lambda r_{\max }, R\right\}$ where $r_{\max }=$ $\inf \left\{r \mid \forall x \in \mathbb{R}^{n}: B_{r}[P-x] \neq \emptyset\right\}, \lambda=(1-\epsilon)^{-1}$. Let $r^{\prime}=r^{\prime}\left(r+\lambda r_{\max }\right)$ according to Corollary 4.17 .

Suppose $B_{r^{\prime}+\lambda r_{\max }}[\varphi(P)-\varphi(x)]=B_{r^{\prime}+\lambda r_{\max }}[\varphi(P)-\varphi(y)]$. Choose $h \in \varphi(P)-\varphi(x)$ such that $|h| \leq \lambda r_{\max }$. Let $x^{\prime}=\varphi^{-1}(\varphi(x)+h)$ and $y^{\prime}=\varphi^{-1}(\varphi(y)+h)$. Then $\varphi\left(x^{\prime}\right), \varphi\left(y^{\prime}\right) \in \varphi(P)$ and $B_{r^{\prime}}[\varphi(P)-$ $\left.\varphi\left(x^{\prime}\right)\right]=B_{r^{\prime}}\left[\varphi(P)-\varphi\left(y^{\prime}\right)\right]$. From Corollary 4.17 we conclude that $B_{r+\lambda r_{\max }}\left[P-x^{\prime}\right]=B_{r+\lambda r_{\max }}\left[P-y^{\prime}\right]$. Since $d \varphi$ is $P$-equivariant with range $r$ the latter implies that $\varphi(x)-\varphi\left(x^{\prime}\right)=\varphi\left(y^{\prime}+x-x^{\prime}\right)-\varphi\left(y^{\prime}\right)$ which, in turn, is equivalent to $\varphi(y)=\varphi\left(y^{\prime}+x-x^{\prime}\right)$. Since $\varphi$ is invertible we get $y-y^{\prime}=x-x^{\prime}$ and hence $B_{r}[P-x]=B_{r}[P-y]$.

Corollary 4.19. Let $P$ be a Delone set of finite local complexity. There exists $1>\epsilon>0$ such that for all differentiable functions $\varphi: \mathbb{R}^{n} \rightarrow$ $\mathbb{R}^{n}$ whose differential satisfies $\|d(\varphi-\mathrm{id})\|_{\infty}<\epsilon$ and is strongly $P$ equivariant we also have that $d \varphi^{-1}$ is strongly $\varphi(P)$-equivariant.

Proof. Given $P$ there exists $\epsilon$ according to the last theorem. Suppose $\|d(\varphi-\mathrm{id})\|_{\infty}<\epsilon$ and that $d \varphi$ is $P$-equivariant with range $r$. Let $r^{\prime}$ correspond to the one needed in the last theorem. Let $B_{r^{\prime}}(\varphi(P)-$ $\varphi(x))=B_{r^{\prime}}(\varphi(P)-\varphi(y))$. By the last theorem this implies $B_{r}(P-x)=$ $B_{r}[P-y]$. Hence $d \varphi(x)=d \varphi(y)$. Since

$$
\left(d \varphi^{-1}\right)(\varphi(x))=(d \varphi(x))^{-1}
$$

(the inverse in the matrix sense) we see that $d \varphi^{-1}$ is $P$-equivariant with range $r^{\prime}$.

\footnotetext{
${ }^{3}$ The argument brakes down if one allows arbitrary $x \in \mathbb{R}^{n}$ as replacing in the definition of $A_{P}$ the union over $x \in P$ by a union over $x \in \mathbb{R}^{n}$ would yield $A_{P}(s)=0$.
} 
We can now answer the question what it means if two closed forms differ by an exact form, at least for forms which are close to did.

Corollary 4.20. Let $P$ be a Delone set of finite local complexity. There exists $1>\epsilon>0$ such that if $d \varphi$ and $d \psi$ are strongly $P$-equivariant, $\|d(\varphi-\mathrm{id})\|_{\infty}<\epsilon$, and furthermore $\varphi-\psi$ weakly $P$-equivariant (or even strongly $P$-equivariant) then $(\psi-\varphi) \circ \varphi^{-1}$ is weakly $\varphi(P)$-equivariant (or even strongly $\varphi(P)$-equivariant). In particular, $\psi \circ \varphi^{-1}$ induces a topological semi-conjugacy between $\left(\Omega_{\varphi(P)}, \mathbb{R}^{n}\right)$ and $\left(\Omega_{\psi(P)}, \mathbb{R}^{n}\right)$ (or even $\psi(P)$ is locally derivable from $\varphi(P))$.

Proof. Suppose $\varphi-\psi$ is $P$-equivariant with range $r$. By Theorem 4.18 we find $r^{\prime}$ such that $B_{r^{\prime}}[\varphi(P)-x]=B_{r^{\prime}}[\varphi(P)-y]$ implies $B_{r}[P-$ $\left.\varphi^{-1}(x)\right]=B_{r}\left[P-\varphi^{-1}(x)\right]$. Since $\eta(x)=(\varphi-\psi)\left(\varphi^{-1}(x)\right)$ the latter implies $\eta(x)=\eta(y)$. Hence $\eta$ is $P$-equivariant with range $r^{\prime}$ and so the result follows directly from Lemma 4.2 (the $\varphi, g$ and $P$ there correspond to id $-\eta$, id and $\varphi(P)$ here.)

4.4. Locally deriving back. When are $\varphi(P)$ and $P$ mutually locally derivable? Suppose that $\varphi-$ id is strongly $P$-equivariant so that in particular $\varphi(P)$ is locally derivable from $P$. The last corollary gives an answer to the question under which condition $P$ can be locally derived back from $\varphi(P)$, namely if $\|d(\varphi-\mathrm{id})\|_{\infty}<\epsilon$ for some $\epsilon$ whose size could be estimated using the details of Corollary 4.17.

4.5. Cohomological interpretation. We interprete our results in cohomological terms.

Let $B_{\|\cdot\|_{\infty}}(\alpha, \epsilon)$ be the open $\epsilon$-ball around $\alpha \in Z_{s-P}^{1}\left(\mathbb{R}^{n}, \mathbb{R}^{n}\right)$ w.r.t. the $\|\cdot\|_{\infty}$-norm. A good measure for the size of the deformation defined by $\varphi$ is given by $\inf \left\{\|d \varphi+d \eta\|_{\infty}: d \eta \in N_{s-P}^{1}\left(\mathbb{R}^{n}, \mathbb{R}^{n}\right)\right\}$. It is scale invariant and corresponds to the maximal relative change of distance between points resulting from the deformation.

Theorem 4.8 and Corollaries 4.19, 4.20 allow for the following interpretation. For all Delone sets of finite local complexity there exists an $\epsilon$ such that the elements of $Z_{s-P}^{1}\left(\mathbb{R}^{n}, \mathbb{R}^{n}\right) \cap B_{\|\cdot\|_{\infty}}(d$ id, $\epsilon)$ define invertible deformations of $P$. If moreover two such elements differ by an element of $B_{w-P}^{1}\left(\mathbb{R}^{n}, \mathbb{R}^{n}\right)$ (or even $B_{s-P}^{1}\left(\mathbb{R}^{n}, \mathbb{R}^{n}\right)$ ) then they define pointed topological conjugate (or even mutually locally derivable) deformations.

Theorems 4.13, 4.15] say that the elements near the class of did in the mixed cohomology group $Z_{s-P}^{1}\left(\mathbb{R}^{n}, \mathbb{R}^{n}\right) / B_{w-P}^{1}\left(\mathbb{R}^{n}, \mathbb{R}^{n}\right) \cap Z_{s-P}^{1}\left(\mathbb{R}^{n}, \mathbb{R}^{n}\right)$ parametrise small deformations modulo bounded deformations which are in the same pointed conjugacy class. Here we say that a deformation defined by a map $\varphi$ is bounded if $\varphi$-id is bounded, a condition which implies that the deformation is homotopic to the original pattern in 
the Hausdorff metric. Note that a bounded deformation has finite size but the converse need not be true.

On the level of pattern spaces and their associated dynamical systems the following picture has emerged (Theorem 4.10). An element $d \varphi$ near did of $Z_{s-P}^{1}\left(\mathbb{R}^{n}, \mathbb{R}^{n}\right)$ defines a homeomorphism from the hull of $P$ to that of the deformation it defines which restricts to a homeomorphism between the canonical transversals and maps $P$ to its deformation. If $d(\varphi-$ id $)$ lies even in $B_{w-P}^{1}\left(\mathbb{R}^{n}, \mathbb{R}^{n}\right) \cap Z_{s-P}^{1}\left(\mathbb{R}^{n}, \mathbb{R}^{n}\right)$ then it defines a second homeomorphism, this time a pointed conjugacy between the continuous dynamical systems. The two homeomorphisms do in general not coincide.

\section{REFERENCES}

[BSJ91] M. Baake, M. Schlottmann, and P.D. Jarvis, Quasiperiodic tilings with tenfold symmetry and equivalence with respect to local derivability, J. Phys. A 24, 4637-4654, (1991).

[CS06] A. Clark, L. Sadun. When Shape Matters: Deformations of Tiling Spaces. Ergod. Th. \& Dyn. Systems 26, 69-86 (2006).

[G] F. Gähler. Talk given at the conference "Aperiodic Order, Dynamical Systems, Operator Algebras and Topology" 2002, slides available at: www.pims.math.ca/science/2002/adot/lectnotes/Gaehler

[Ke97] J. Kellendonk. Topological equivalence of tilings. Journal of Mathematical Physics 38, 1823-1842, (1997).

[Ke03] J. Kellendonk. Pattern-equivariant functions and cohomology. J. Phys. A $36,1-8(2003)$.

[KR06] J. Kellendonk, S. Richard, Topological boundary maps in physics: General theory and applications. preprint math-ph/0605048.

[KP06] J. Kellendonk, I. F. Putnam. The Ruelle-Sullivan map for actions of $\mathbb{R}^{n}$, Math. Ann. 334, 693-711 (2006).

[Sa03] L. Sadun. Tiling Spaces are Inverse Limits. J. of Math. Phys. 44, 5410-5414 (2003).

[Sadun] L. Sadun. Pattern-Equivariant Cohomology with Integer Coefficients. Preprint.

[SW03] L. Sadun, R.F. Williams Tiling Spaces are Cantor Set Fiber Bundles. Erg. Th. \& Dyn. Systems 23, 307-316, (2003).

Universit de Lyon, Universit Lyon1, CNRS, UMR 5208 Institut Camille Jordan, Batiment du Doyen Jean Braconnier, 43, Blvd du 11 novembre 1918, F - 69622 Villeurbanne Cedex, France 